Nonequilibrium transport through a kondo dot in magnetic field perturbation theory

Paaske, Jens; Rosch, A.; Wolfle, P.

Published in:

Physical Review B Condensed Matter

DOI:

10.1103/PhysRevB.69.155330

Publication date:

2004

Document version

Early version, also known as pre-print

Citation for published version (APA):

Paaske, J., Rosch, A., \& Wolfle, P. (2004). Nonequilibrium transport through a kondo dot in magnetic field: perturbation theory. Physical Review B Condensed Matter, 69(15), 155330.

https://doi.org/10.1103/PhysRevB.69.155330 


\title{
Nonequilibrium transport through a Kondo dot in a magnetic field: Perturbation theory
}

\author{
J. Paaske, A. Rosch, and P. Wölfle \\ Institut für Theorie der Kondensierten Materie, Universität Karlsruhe, D-76128 Karlsruhe, Germany
}

(Received 15 July 2003; revised manuscript received 8 October 2003; published 28 April 2004)

\begin{abstract}
Using nonequilibrium perturbation theory, we investigate the nonlinear transport through a quantum dot in the Kondo regime in the presence of a magnetic field. We calculate the leading logarithmic corrections to the local magnetization and the differential conductance, which are characteristic of the Kondo effect out of equilibrium. By solving a quantum Boltzmann equation, we determine the nonequilibrium magnetization on the dot and show that the application of both a finite bias voltage and a magnetic field induces a novel structure of logarithmic corrections not present in equilibrium. These corrections lead to more pronounced features in the conductance, and their form calls for a modification of the perturbative renormalization group.
\end{abstract}

DOI: 10.1103/PhysRevB.69.155330

PACS number(s): 73.63.Kv, 72.10.Fk, 72.15.Qm

A localized spin coupled to the spins of a conductionelectron system via a Heisenberg exchange interaction is known to give rise to the Kondo effect, provided the coupling is antiferromagnetic. ${ }^{1}$ At sufficiently high temperatures or energies, well above the characteristic energy scale referred to as the Kondo temperature $T_{K}$ the signature of the Kondo effect is a logarithmic variation of various observables with temperature (or other energies). As first demonstrated by Kondo, ${ }^{2}$ such logarithmic behavior appears already in perturbation theory to low order in the exchange coupling. At low temperatures, however, for $T \ll T_{K}$, the local spin is screened by the conduction-electron spins, and the system enters a local Fermi-liquid state, characterized by integer temperature power laws.

The many-body resonance state forming near $T_{K}$ comprises infinitely many virtual particle-hole excitations leading to a peak in the conduction-electron-scattering amplitude at the Fermi energy: the Kondo resonance. In equilibrium systems the Bethe ansatz method allows us to analytically calculate thermodynamic properties, ${ }^{3}$ and dynamical properties can be determined with the help of Wilson's numerical renormalization group. ${ }^{4}$ These methods reveal the universal nature of the Kondo effect: a single characteristic scale $T_{K}$ determines the physics and all physical quantities are universal functions of, e.g., $T / T_{K}$. Already in 1970 Anderson $^{5}$ suggested a simple and efficient method, known as "poor man's scaling" or perturbative renormalization group, to resum the leading logarithmic terms in perturbation theory and to establish the scaling behavior. It requires nothing more than low-order perturbation theory, and provides a controlled approximation for $T>T_{K}$, i.e., as long as the running exchange couplings remain small.

While the Kondo effect was discovered in metals containing magnetic impurities in the 1960s (with experimental observations dating back to the 1930s), and most of the theoretical development took place before the mid 1980s, it had a revival in the $1990 \mathrm{~s}$ in the context of electron transport through quantum dots weakly coupled to leads. Provided that the dot carries a net spin, a Kondo resonance develops, thus admitting resonant tunneling of electrons. ${ }^{6,7}$ This leads to a removal of the Coulomb blockade, i.e., an increase of the conductance from small values up to the quantum limit, as the Kondo resonance develops. We shall refer to this type of quantum dot as a Kondo dot. It has been observed that the Kondo effect is quenched by raising the transport bias voltage $V$ well above $T_{K}$, i.e., $e V \gg T_{K}$, and that the presence of a magnetic field splits the zero-bias conductance peak into two distinct peaks, located at bias voltages roughly equal to plus and minus the Zeeman splitting of the spin on the dot. $^{8-12}$

The observation of Kondoesque conductance anomalies, exhibiting a logarithmic temperature dependence of the zerobias peak height and a Zeeman splitting of the peak in finite magnetic field, actually has a much longer history; it has often been observed in more traditional tunnel junctions involving tunneling via magnetic impurities. In metalinsulator-metal junctions the Kondo anomaly can result from magnetic impurities present in the insulating metal-oxide barrier, from surface states at the metal/metal-oxide interface $^{13-17}$ or even from unpaired electrons residing on organic radicals in a polymerized benzene barrier. ${ }^{18}$ The same type of conductance anomaly has been detected in semiconductor-metal (Schottky) junctions, ${ }^{19,20}$ in which the neutral shallow donors in the semiconductor depletion layer provide the spin-1/2 moments which incite the Kondo correlations.

In 1966, Appelbaum ${ }^{21,22}$ and Anderson ${ }^{23}$ demonstrated that this type of conductance anomaly can result from socalled exchange tunneling processes, in which an electron tunnels from one electrode to the other via an intermediate magnetic impurity orbital and at the same time flips its spin. This mechanism was shown to lead to a Kondo effect enhancing the charge transfer across the dot, and it explains in a natural way why the conductance is peaked at a bias voltage corresponding to the Zeeman splitting of the impurity moment: the finite bias has to supply the energy to flip the spin in the presence of a magnetic field. Appelbaum calculated the tunneling conductance from a simple golden rule expression, in which the tunneling amplitudes were determined from third-order perturbation theory, including the leading logarithmic corrections reflecting the Kondo effect. His result did capture the qualitative features of the conductance anomaly, but any quantitative agreement with experiment has been restricted to the case of zero magnetic field. Wolf and Losee ${ }^{24}$ later suggested an extension of Appelbaum's theory in which the logarithmic enhancement was cut 
off by the lifetime broadening of the Zeeman split spin levels. Indeed this extra feature improved the agreement with experiments somewhat, but even with several parameters, this did not allow for a fit to experiments at finite magnetic field. ${ }^{16-19,25}$

The mechanism of exchange tunneling, suggested by Appelbaum and Anderson, was originally built into a Hamiltonian with constant, a priori unknown, exchange couplings to the spin. While this approach relied on an exchange tunneling term connecting the two electrodes [see the model (1) below], a parallel development by Sólyom and Zawadowski ${ }^{26}$ suggested that the conductance anomaly should arise from the energy dependent renormalization of the local density of states, induced by Kondo spins near one of the electrodes not directly coupled to the other side. Initially the two proposals disagreed even in the sign of the change in conductance, but these differences were resolved in a later work by Appelbaum and Brinkman ${ }^{27}$ using Zawadowski's alternative approach to tunneling. ${ }^{28,29}$ The final reconciliation of these ideas came with the work of Ivezić, ${ }^{30}$ taking the nonequilibrium Keldysh approach to tunneling, developed earlier by Caroli et al. ${ }^{31}$ The fact that the electrodes were out of mutual equilibrium was taken into account, and it was demonstrated that the earlier (essentially equilibrium) treatments ${ }^{26,27}$ were only correct in cases when the impurity was located near one of the electrodes, whereas an impurity somewhere in the middle of the barrier constitutes a true nonequilibrium problem. For a review of these earlier works cf. also Ref. 32.

With the discovery of the Kondoesque tunneling anomaly in various types of quantum dots, this venerable problem has recently been revived. Meanwhile, experimenting with quantum dots offers much better control over the parameters defining the problem, and this in turn allows for a more systematic study of the physics underlying the conductance anomaly. Since the zero-bias anomaly arises only in Coulomb blockade valleys corresponding to an odd number of electrons occupying the dot, the effective local moment can be ascribed to a single electron in the uppermost energy level. In this way the "magnetic impurity" spreads over the entire dot and there is no confusion as to whether the impurity is located close to, or even residing in, one of the electrodes or whether one should average over many impurities, issues which were all very important in the conventional tunnel junctions mentioned above. In particular, there is no reason to believe that the dot spin should be equilibrated with one particular lead, which was pointed out by Ivezic ${ }^{31}$ to constitute a true nonequilibrium problem. Within the Anderson model, this problem has been studied using an equations-of-motion technique combined with the noncrossing approximation ${ }^{33-38}$ and by means of nonequilibrium perturbation theory, expanding in the hybridization strength. ${ }^{39}$ Other works have taken the Kondo model as their starting point, but have mostly focused on the effects of an applied ac bias in the case of zero magnetic field. ${ }^{40,41}$

A complete theory of the Kondo dot in a nonequilibrium stationary state, i.e., in the presence of a finite current flowing through the dot, does not exist yet. Most of the methods which have proven so successful in dealing with the equilib- rium Kondo problem, appear to have no trivial extension to a nonequilibrium situation. Schiller and co-workers ${ }^{42}$ have successfully applied bosonization techniques, originally devised by Emery and Kivelson ${ }^{43}$ in their solution of the twochannel Kondo problem, to calculate a number of observables near a certain Toulouse point in the presence of a finite bias voltage. It is unclear, however, to what extent these results apply to the generic Kondo model. Konik, Saleur and Ludwig $^{44}$ started from the Bethe ansatz solution in equilibrium to construct approximate scattering states, in the presence of a finite voltage. To what extent their approximations and boundary conditions are valid is not obvious to us. To the best of our knowledge, even the perturbation theory in a true nonequilibrium situation has not yet been worked out in the presence of a magnetic field to leading logarithmic order, i.e., to third order in the exchange interactions. This latter task will be taken on in the present paper, which presents a detailed analysis of the nonequilibrium perturbation theory to leading logarithmic order, generalizing Appelbaum's result to the case where the spin is not equilibrated with one of the leads.

Our aim here is to understand the physical processes governing the nonequilibrium situation and to formulate a starting point for the resummation of the leading logarithmic terms in perturbation theory. Even in the perturbative regime, when magnetic fields, voltages, or temperatures are large compared to $T_{K}$, such a resummation is necessary not only to recover the correct universal scaling behavior but also to be able to fit experiments quantitatively. ${ }^{45}$ In some of the early works ${ }^{26,46}$ perturbation theory was resummed using approximations of Nagaoka, ${ }^{47}$ Abrikosov, ${ }^{48}$ or Suhl and Wong, ${ }^{49}$ mainly as an attempt to capture the strong-coupling regime. As for the quantum dots, Kaminski et al. ${ }^{41}$ first suggested a poor man's scaling method to deal with this problem at finite voltage, but this approach did not encompass the case of a finite magnetic field and therefore avoided the conceptual problem connected to the calculation of the magnetization.

Recently, the present authors suggested a different poor man's scaling approach, ${ }^{45}$ which effectively resums the logarithmic corrections and recovers scaling, even in the presence of a magnetic field and a finite voltage. As an important check on this approach, it was crucial that an expansion in bare parameters would correctly reproduce the leading logarithmic corrections found from perturbation theory, and indeed this was found to be the case. Main results of the nonequilibrium perturbation theory presented here were briefly stated in Ref. 45. The details of our scaling approach will be provided in a subsequent publication. ${ }^{50}$

The remaining parts of the paper are organized as follows. In Sec. I we define our model and briefly review the pseudofermion formalism to be used throughout the paper. Section II reviews the diagrammatic rules of nonequilibrium perturbation theory using the Keldysh formalism, and introduces a few basic building blocks from which all subsequent diagrams will be constructed. In Sec. III we solve a quantum Boltzmann equation to find the nonequilibrium distribution function for the local spin, and demonstrate that this leads to rather dramatic effects of the bias voltage on the local mag- 


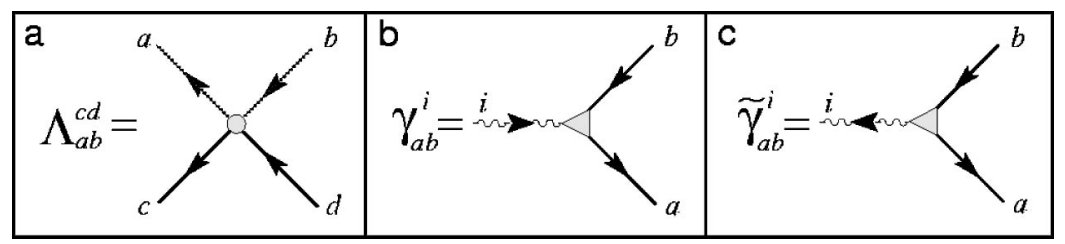

FIG. 1. (a) The bare conduction-electronpseudofermion vertex. (b) The absorption and (c) the emission vertices. Propagators are dashed $(p f)$ and full (ce) lines. Note that the absorption and emission vertices could equally well have been drawn with $p f$ propagators.

$$
\langle\mathcal{O}\rangle_{Q=1}=\lim _{\lambda \rightarrow \infty} \frac{\langle\mathcal{O} Q\rangle_{\lambda}}{\langle Q\rangle_{\lambda}}
$$

tunneling current which takes the nonequilibrium magnetization as its input. Section V contains a summary and discussion of our results.

\section{THE MODEL}

We consider the Hamiltonian

$$
\begin{aligned}
H= & \sum_{\alpha, \mathbf{k}, \sigma}\left(\varepsilon_{\mathbf{k}}-\mu_{\alpha}\right) c_{\alpha \mathbf{k} \sigma}^{\dagger} c_{\alpha \mathbf{k} \sigma}-g \mu_{B} B S_{z} \\
& +\sum_{\alpha, \alpha^{\prime}, \mathbf{k}, \mathbf{k}^{\prime}, \sigma, \sigma^{\prime}} J_{\alpha^{\prime} \alpha} \vec{S} \cdot \frac{1}{2} c_{\alpha^{\prime} \mathbf{k}^{\prime} \sigma^{\prime}}^{\dagger} \vec{\tau}_{\sigma^{\prime} \sigma} c_{\alpha \mathbf{k} \sigma},
\end{aligned}
$$

where $\mu_{L, R}= \pm e V / 2, S_{z}$ is the spin-1/2 on the dot, and $\vec{\tau}$ the Pauli matrices. We shall use the dimensionless coupling constants $g_{i}=N(0) J_{i}$, where $N(0)$ is the density of states per spin for the conduction electrons and $J_{L L}, J_{R R}$, and $J_{L R}$ $=J_{R L}$ the real-valued exchange constants. For simple quantum dots in the Kondo regime, which can be described by an Anderson model, the exchange coupling constants are related by $g_{L R}^{2}=g_{L L} g_{R R}$. However, in more complex situations, such as in double dot systems in the Kondo regime, no such relations exist and we will therefore treat $g_{L R}$ as an independent parameter of our Hamiltonian (1). For notational convenience we employ the shorthand notation $g_{d}=\left(g_{L L}\right.$ $\left.+g_{R R}\right) / 2$ and $g^{2}=\left(g_{L L}^{2}+g_{R R}^{2}+2 g_{L R}^{2}\right) / 4$, and unless specifically stated otherwise, we will henceforth use units where $\hbar=k_{B}=g \mu_{B}=e=1$.

In order to proceed with a perturbative calculation, which includes also the effects of a magnetic field, it is convenient to apply a fermionic representation of the local spin operators. We choose here Abrikosov's ${ }^{48}$ pseudofermion representation, which, in terms of fermionic charge-neutral spin-1/2 operators $f$, reads

$$
\vec{S}=\frac{1}{2} \sum_{\gamma \gamma^{\prime}} f_{\gamma}^{\dagger} \vec{\tau}_{\gamma \gamma^{\prime}} f_{\gamma^{\prime}}
$$

Since only the singly occupied fermion states have any physical relevance, this representation must be supplemented by a projection onto this physical part of the Hilbert space, effectively excluding doubly occupied and empty states. To this end, the pseudofermion is endowed with a chemical potential $\lambda$, which is kept finite throughout the calculation, using a grand-canonical ensemble average. As demonstrated in Ref. 51, the physically relevant, i.e., canonical ensemble averaged, expectation value of an observable $\mathcal{O}$ is obtained as the limiting value where $Q=\Sigma_{\gamma} f_{\gamma}^{\dagger} f_{\gamma}$ is the pseudofermion number operator. Note that in the common case, where the observable $\mathcal{O}$ has zero expectation value in the $Q=0$ ensemble, one may leave out the $Q$ operator from the numerator in Eq. (3). This procedure applies equally well within the Keldysh formulation of nonequilibrium perturbation theory, since the statistical averaging in this approach is performed with respect to a thermal equilibrium state in the infinite past. A similar projection technique was used in Ref. 34, and later in Ref. 35, to study the Anderson model out of equilibrium.

Since $\lambda$ enters as a chemical potential, thermal averages taken with a finite $\lambda$ contain various powers of $e^{-\lambda / T}$. In particular, $\langle Q\rangle_{\lambda} \sim e^{-\lambda / T}$ and therefore the limit of $\lambda \rightarrow \infty$ in Eq. (3) effectively picks out the terms in $\langle\mathcal{O} Q\rangle_{\lambda}$ which are also proportional to $e^{-\lambda / T}$. Likewise, in calculating any thermal average of interest at finite $\lambda$, one is allowed to retain only the terms of lowest order in $e^{-\lambda / T}$.

\section{KELDYSH DIAGRAMMATICS}

In setting up the nonequilibrium perturbation theory, we shall comply with the conventions in Ref. 52. All Keldyshspace matrix propagators are represented in the usual upperright triangular form

$$
\underline{G}=\left(\begin{array}{cc}
G^{R} & G^{K} \\
0 & G^{A}
\end{array}\right)
$$

and the individual entries will be denoted by latin indices. From this basic Green function, one may obtain the usual spectral, lesser, and greater functions as

$$
\begin{gathered}
A=i\left(G^{R}-G^{A}\right), \\
G^{<}=\left(G^{K}-G^{R}+G^{A}\right) / 2, \\
G^{>}=\left(G^{K}+G^{R}-G^{A}\right) / 2, \\
\operatorname{Re}[G]=\left(G^{R}+G^{A}\right) / 2,
\end{gathered}
$$

implying the $R$ superscript in the real part. A corresponding notation will be used for self-energies, except that the spectral function will be replaced by the broadening $\Gamma=i\left(\Sigma^{R}\right.$ $\left.-\Sigma^{A}\right)$. We shall henceforth denote conduction-electron $(c e)$ and pseudofermion $(p f)$ correlation functions by capital latin and calligraphic letters, respectively.

Perturbation theory in terms of Keldysh matrix propagators involves the bare four-point vertex and the measurement vertices depicted in Fig. 1. These have the tensor structure

$$
\Lambda_{a b}^{c d}=\frac{1}{2}\left(\delta_{a b} \tau_{c d}^{1}+\tau_{a b}^{1} \delta_{c d}\right)
$$




$$
\begin{aligned}
& \gamma_{a b}^{1}=\tilde{\gamma}_{a b}^{2}=\frac{1}{\sqrt{2}} \delta_{a b}, \\
& \gamma_{a b}^{2}=\tilde{\gamma}_{a b}^{1}=\frac{1}{\sqrt{2}} \tau_{a b}^{1} .
\end{aligned}
$$

Diagrams should be interpreted with the usual zerotemperature Feynman rules, including a prefactor $(i J)^{n}(-1)^{\mathrm{F}_{p f}}+\mathrm{F}_{c e}$ to any diagram of order $J^{n}$, having $F_{p f, c e}$ closed pseudofermion or conduction-electron loops. Both flavors propagate with spin indices $\gamma, \sigma= \pm 1$, to which we apply the convention that $\bar{\gamma} \equiv-\gamma$. Furthermore, the conduction-electron Green functions carry a lead index $\alpha$ $\in\{L, R\}$. The Einstein summation convention is implied for all indices except where otherwise stated.

Since we assume the total system to have reached a steady state, all Green functions depend only on one frequency. The bare $p f$ propagator has the spectral function

$$
\mathcal{A}_{\gamma}(\omega)=2 \pi \delta(\omega+\gamma B / 2)
$$

and corresponding retarded and advanced Green functions. For the Keldysh component, we make the usual ansatz that

$$
\mathcal{G}_{\gamma}^{K}(\omega)=i \mathcal{A}_{\gamma}(\omega)\left[2 n_{\gamma \lambda}(\omega)-1\right] \text {, }
$$

which defines the $p f$ distribution function $n_{\gamma \lambda}(\omega)$. At zerobias voltage, where the spin is equilibrated with the conduction electrons, $n_{\gamma \lambda}(\omega)$ reduces to the Fermi function $f(\omega$ $+\lambda)=1 /\left(e^{(\omega+\lambda) / T}+1\right)$. We follow the convention of Ref. 53 and place the chemical potentials in the distribution functions rather than in the spectral functions, which prevents the parameter $\lambda$ from pervading the formulas. The unprojected mean occupation numbers are given by the integral

$$
n_{\gamma \lambda}=\int_{-\infty}^{\infty} \frac{d \omega}{2 \pi} \mathcal{A}_{\gamma}(\omega) n_{\gamma \lambda}(\omega)
$$

that is, $n_{\gamma \lambda}=n_{\gamma \lambda}(-\gamma B / 2)$ for bare $\mathcal{A}_{\gamma}$. After taking the limit $\lambda \rightarrow \infty$ for a given observable and thus performing the projection onto the physical spin states, one can use the constraint $n_{\uparrow}+n_{\downarrow}=1$ to write the local magnetization as $M$ $=2 n_{\uparrow}-1$. At times, we shall also use the shorthand $M_{\gamma, \lambda}$ $=2 n_{\gamma, \lambda}-1$ prior to projection.

The bare conduction-electron Green functions depend on momentum as well as frequency, but since the interaction and the pseudofermions are local in space, all internal lines in a Feynman diagram involve only propagation in time, and we are allowed to work with local, momentum integrated, $c e$ Green functions. Assuming a constant density of states in a band of width $2 D$, centered at zero, the momentum integrated spectral function reads

$$
A(\omega)=2 \pi N(0) \Theta(D-|\omega|),
$$

with $N(0)=1 /(2 D)$. Assuming the electrons in separate leads to be in thermal equilibrium, the lead-dependent Keldysh Green function takes the form

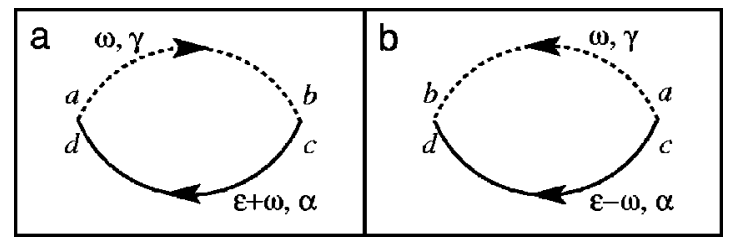

FIG. 2. The conduction-electron-pseudofermion bubbles in Peierls (a) and Cooper (b) channels. Conduction-electron propagators have lead index $\alpha$ and pseudofermion propagators carry the spin index $\gamma$. Latin indices refer to the Keldysh-matrix structure in Eq. (4).

$$
G_{\alpha}^{K}(\omega)=-i A(\omega) \tanh \left(\frac{\omega-\mu_{\alpha}}{2 T}\right),
$$

in terms of the chemical potentials $\mu_{L}=-\mu_{R}=V / 2$. The spectral function (15) implies a small real part $\operatorname{Re}\left[G^{R}(\omega)\right]$ $=(N(0) / 2) \ln [(\omega+D) /(\omega-D)] \approx N(0) \omega / D$ which we can safely neglect for most of our discussion.

\section{A. Second-order vertex functions}

The central objects in the diagrammatics of this problem are the ce-pf bubbles depicted in Fig. 2, insofar as they carry the logarithmic integrals which eventually lead to the Kondo effect. The bubbles in the Peierls and Cooper channels are evaluated as

$$
\begin{aligned}
& { }_{\gamma}^{\alpha} \mathcal{K}_{b a}^{d c}(\varepsilon)=\int \frac{d \omega}{2 \pi} \underline{G}_{\alpha}^{d c}(\omega+\varepsilon) \underline{\mathcal{G}}_{\gamma}^{b a}(\omega), \\
& { }_{\gamma}^{\alpha} \overline{\mathcal{K}}_{b a}^{d c}(\varepsilon)=\int \frac{d \omega}{2 \pi} \underline{G}_{\alpha}^{d c}(\varepsilon-\omega) \underline{\mathcal{G}}_{\gamma}^{b a}(\omega),
\end{aligned}
$$

from which one can readily determine the various Keldysh components by straightforward integrations:

$$
\begin{gathered}
{ }_{\gamma} \mathcal{K}_{R}^{R}(\varepsilon)={ }_{\gamma} \mathcal{K}_{A}^{A}(\varepsilon)=0, \\
{ }_{\gamma} \mathcal{K}_{A}^{R}(\varepsilon)=i G^{R}(\varepsilon-\gamma B / 2), \\
{ }_{\gamma} \mathcal{K}_{R}^{A}(\varepsilon)=-i G^{A}(\varepsilon-\gamma B / 2), \\
{ }_{\gamma}^{\alpha} \mathcal{K}_{K}^{R, A, K}(\varepsilon)=i G_{\alpha}^{R, A, K}(\varepsilon-\gamma B / 2) M_{\gamma, \lambda}, \\
{ }_{\gamma}^{\alpha} \mathcal{K}_{R}^{K}(\varepsilon)=-\frac{i}{2} G_{\alpha}^{K}(\varepsilon-\gamma B / 2) \\
-i N(0) \ln \left(\frac{D^{2}}{\left(\varepsilon-\mu_{\alpha}-\gamma B / 2\right)^{2}+T^{2}}\right), \\
{ }_{\gamma}^{\alpha} \mathcal{K}_{A}^{K}(\varepsilon)=\frac{i}{2} G_{\alpha}^{K}(\varepsilon-\gamma B / 2) \\
-i N(0) \ln \left(\frac{D^{2}}{\left(\varepsilon-\mu \mu_{\alpha}-\gamma B / 2\right)^{2}+T^{2}}\right),
\end{gathered}
$$

for the Peierls channel, from which the bubbles in the Cooper channel are obtained as 


$$
\begin{gathered}
{ }_{\gamma} \overline{\mathcal{K}}_{A}^{R}(\varepsilon)={ }_{\gamma} \overline{\mathcal{K}}_{R}^{A}(\varepsilon)=0, \\
{ }_{\gamma} \overline{\mathcal{K}}_{R, A}^{R, A}(\varepsilon)=-{ }_{\gamma} \mathcal{K}_{R, A}^{A, R}(-\varepsilon), \\
{ }_{\gamma}^{\alpha} \overline{\mathcal{K}}_{K}^{R, A, K}(\varepsilon)=-{ }_{\gamma}{ }^{\alpha} \mathcal{K}_{K}^{A, R, K}(-\varepsilon), \\
{ }_{\gamma}^{\alpha} \overline{\mathcal{K}}_{R, A}^{K}(\varepsilon)=-{ }_{\gamma}{ }^{\alpha} \mathcal{K}_{R, A}^{K}(-\varepsilon) .
\end{gathered}
$$

In both channels the Kondo effect derives from the imaginary part of bubbles with a $K$-component conduction electron together with a retarded or an advanced pseudofermion. In such combinations, $\operatorname{Re}\left[\mathcal{G}_{\gamma}(\omega)\right] \propto 1 / \omega$ is convoluted with $\tanh (\varepsilon+\omega / 2 T)$ to produce the logarithmic enhancement.

To work out higher-order Feynman diagrams, it is convenient to construct the second-order renormalized vertex functions, obtained by attaching a Keldysh vertex (9) to each end of these bubbles:

$$
\begin{aligned}
& { }_{\gamma}^{\alpha} \underline{\mathcal{I}}_{a b}^{c d}(\varepsilon)=\Lambda_{a b^{\prime} \gamma}^{c^{\prime} d \alpha} \underline{\mathcal{K}}_{b^{\prime} a^{\prime}}^{d^{\prime} c^{\prime}}(\varepsilon) \Lambda_{a^{\prime} b}^{c d^{\prime}}, \\
& { }_{\gamma}^{\alpha} \overline{\mathcal{I}}_{a b}^{d}(\varepsilon)=\Lambda_{a b^{\prime} \gamma}^{c d^{\prime} \alpha} \overline{\mathcal{K}}_{b^{\prime} a^{\prime}}^{d^{\prime} c^{\prime}}(\varepsilon) \Lambda_{a^{\prime} b}^{c^{\prime} d} .
\end{aligned}
$$

Working out the contraction of Keldysh indices one may organize all entries in the Peierls channel as

$$
\begin{gathered}
\underline{\mathcal{I}}_{11}^{12}=\underline{\mathcal{I}}_{12}^{22}=\underline{\mathcal{I}}_{21}^{11}=\underline{\mathcal{I}}_{22}^{21}=\frac{1}{4 i} \mathcal{I}^{R}, \\
\underline{\mathcal{I}}_{12}^{11}=\underline{\mathcal{I}}_{22}^{12}=\underline{\mathcal{I}}_{21}^{22}=\underline{\mathcal{I}}_{11}^{21}=\frac{1}{4 i} \mathcal{I}^{A}, \\
\underline{\mathcal{I}}_{11}^{11}=\underline{\mathcal{I}}_{22}^{22}=\underline{\mathcal{I}}_{21}^{12}=\underline{\mathcal{I}}_{12}^{21}=\frac{1}{4 i} \mathcal{I}^{K}, \\
\underline{\mathcal{I}}_{22}^{11}=\underline{\mathcal{I}}_{11}^{22}=\underline{\mathcal{I}}_{12}^{12}=\underline{\mathcal{I}}_{21}^{21}=0,
\end{gathered}
$$

satisfying the symmetry $\underline{\mathcal{I}}_{a b}^{c d}=\underline{\mathcal{I}}_{\bar{a} b}^{c \bar{d}}=\underline{\mathcal{I}}_{a \bar{b}}^{\bar{c} d}$, and similarly in the Cooper channel

$$
\begin{gathered}
\underline{\mathcal{I}}_{22}^{12}=\underline{\mathcal{I}}_{12}^{22}=\underline{\mathcal{I}}_{21}^{11}=\overline{\mathcal{I}}_{11}^{21}=\frac{1}{4 i} \overline{\mathcal{I}}^{R}, \\
\underline{\mathcal{I}}_{12}^{11}=\underline{\mathcal{I}}_{11}^{12}=\underline{\mathcal{I}}_{21}^{22}=\overline{\mathcal{I}}_{22}^{21}=\frac{1}{4 i} \overline{\mathcal{I}}^{A}, \\
\underline{\mathcal{I}}_{22}^{11}=\underline{\overline{\mathcal{I}}}_{11}^{22}=\underline{\mathcal{I}}_{21}^{12}=\underline{\mathcal{I}}_{12}^{21}=\frac{1}{4 i} \overline{\mathcal{I}}^{K}, \\
\overline{\mathcal{I}}_{11}^{11}=\underline{\overline{\mathcal{I}}}_{22}^{22}=\underline{\overline{\mathcal{I}}}_{12}^{12}=\underline{\mathcal{I}}_{21}^{21}=0,
\end{gathered}
$$

where $\underline{\overline{\mathcal{I}}}_{a b}^{d}=\underline{\mathcal{I}}_{\bar{a} b}^{\bar{c} d}=\overline{\mathcal{I}}_{a \bar{b}}^{c \bar{d}}$. For clarity, we have temporarily suppressed the variables $\gamma, \alpha$, and $\varepsilon$, and written only the Keldysh indices. Furthermore we have introduced new functions, $\mathcal{I}^{R, A, K}$ and $\overline{\mathcal{I}}^{R, A, K}$, with

$$
\begin{aligned}
& \mathcal{I}^{R, A}=i\left(\mathcal{K}_{K}^{R, A}+\mathcal{K}_{A, R}^{K}\right), \\
& \overline{\mathcal{I}}^{R, A}=i\left(\overline{\mathcal{K}}_{K}^{R, A}+\overline{\mathcal{K}}_{R, A}^{K}\right), \\
& \mathcal{I}^{K}=i\left(\mathcal{K}_{K}^{K}+\mathcal{K}_{A}^{R}+\mathcal{K}_{R}^{A}\right), \\
& \overline{\mathcal{I}}^{K}=i\left(\overline{\mathcal{K}}_{K}^{K}+\overline{\mathcal{K}}_{R}^{R}+\overline{\mathcal{K}}_{A}^{A}\right),
\end{aligned}
$$

satisfying the relation ${ }_{\gamma}^{\alpha} \overline{\mathcal{I}}^{R, A, K}(\varepsilon)=-{ }_{\gamma}^{\bar{\alpha}} \mathcal{I}^{A, R, K}(-\varepsilon)$.

From their definitions, these new functions $\mathcal{I}^{R, A, K}$ are seen to have poles in the lower $(R)$, the upper $(A)$, or both $(K)$ half planes, and to satisfy that $\left[\mathcal{I}^{R}(\varepsilon)\right]^{*}=\mathcal{I}^{A}(\varepsilon)$. To be specific, one finds from Eq. (19),

$$
\begin{aligned}
&{ }_{\gamma}^{\alpha} \mathcal{I}^{R}(\varepsilon)=-M_{\gamma, \lambda} G_{\alpha}^{R}(\varepsilon-\gamma B / 2)-\frac{1}{2} G_{\alpha}^{K}(\varepsilon-\gamma B / 2) \\
&+N(0) \ln \left(\frac{D^{2}}{\left(\varepsilon-\mu_{\alpha}-\gamma B / 2\right)^{2}+T^{2}}\right), \\
&{ }_{\gamma}^{\alpha} \mathcal{I}^{K}(\varepsilon)=i A(\varepsilon-\gamma B / 2)\left[1+M_{\gamma, \lambda} \tanh \left(\frac{\varepsilon-\mu_{\alpha}-\gamma B / 2}{2 T}\right)\right] .
\end{aligned}
$$

The real and the imaginary part of $\mathcal{I}^{R}$ are

$$
\begin{gathered}
\operatorname{Re}\left[{ }_{\gamma}^{\alpha} \mathcal{I}(\varepsilon)\right]= \\
N(0) \ln \left(\frac{D^{2}}{\left(\varepsilon-\mu_{\alpha}-\gamma B / 2\right)^{2}+T^{2}}\right) \\
-M_{\gamma, \lambda} \operatorname{Re}\left[G_{\alpha}(\varepsilon-\gamma B / 2)\right], \\
\operatorname{Im}\left[{ }_{\gamma}^{\alpha} \mathcal{I}(\varepsilon)\right]=\frac{1}{2} A(\varepsilon-\gamma B / 2)\left[M_{\gamma, \lambda}+\tanh \left(\frac{\varepsilon-\mu_{\alpha}-\gamma B / 2}{2 T}\right)\right],
\end{gathered}
$$

while the lesser and greater components take the following form:

$$
\begin{aligned}
& { }_{\gamma}^{\alpha} \mathcal{I}^{<}(\varepsilon)=2 i A(\varepsilon-\gamma B / 2)\left(1-n_{\gamma, \lambda}\right) f\left(\varepsilon-\mu_{\alpha}-\gamma B / 2\right), \\
& { }_{\gamma}^{\alpha} \mathcal{I}^{>}(\varepsilon)=2 i A(\varepsilon-\gamma B / 2) n_{\gamma, \lambda}\left[1-f\left(\varepsilon-\mu_{\alpha}-\gamma B / 2\right)\right] .
\end{aligned}
$$

Note that since $\operatorname{Re}[G(\omega)] \approx N(0) \omega / D$ for $\omega \ll D$, contributions from the last term in Eq. (28), of order $\max [B, V] / D$ $\ll 1$, can safely be neglected.

The real and imaginary parts, (28) and (29), satisfy the Kramers-Kronig relation, and altogether their analytical properties allow us to interpret $\mathcal{I}$ as genuine Keldysh Green functions describing the (time-) parallel or antiparallel propagation of conduction electrons and pseudofermions. The different components $(R, A,<,>)$ of this mixed bubble could have been written down immediately using the Langreth rules $^{54}$ for analytical continuation, and in fact this method is also very convenient for determining the different components of the second-order self-energy. For the third-order self-energy, however, we find the Keldysh matrix structure to be more convenient when dealing with the large number of 


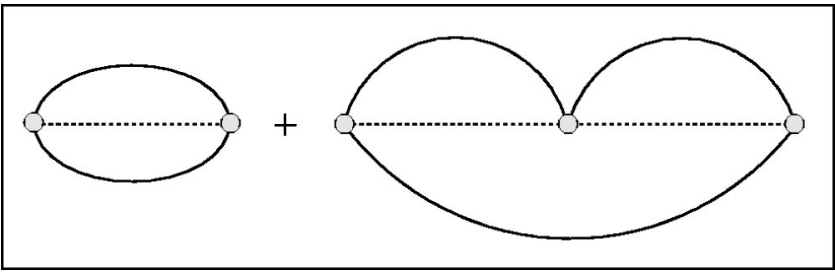

FIG. 3. Unlabeled Feynman diagrams for the $p f$ self-energies.

contractions appearing to this order. The catalogs (23) and (24) have been worked out for this purpose and are used repeatedly in working out the different Keldysh contractions appearing throughout the paper.

\section{NONEQUILIBRIUM MAGNETIZATION}

\section{A. Pseudofermion self-energy}

With these basic diagrammatic objects at hand, we can now readily evaluate the Feynman diagrams for the $p f$ selfenergies shown in Fig. 3. It is, however, essential to realize that the occupation functions $n_{\gamma \lambda}$ on the dot are completely undetermined to zeroth order of perturbation theory, i.e., in the absence of a coupling to the leads. ${ }^{55}$ These are therefore kept as free parameters to be determined later. The first-order $p f$ self-energy vanishes, since we have not included a Zeeman term for the conduction electrons. Nevertheless, the corresponding Hartree term would be entirely real and contribute only by a constant shift of the $p f$ energy levels (cf. Appendix).

\section{Second-order self-energy}

Neglecting the Zeeman term for the conduction electrons, the $a b$ component of the second-order self-energy from Fig. 3 is given as

$$
\underline{\Sigma}_{\gamma \lambda}^{a b(2)}(\omega)=\frac{\theta_{\gamma \gamma^{\prime}}}{8} J_{\alpha \alpha^{\prime}}^{2} \int \frac{d \varepsilon}{2 \pi} \gamma^{\prime} \underline{\mathcal{I}}_{a b}^{c d}(\varepsilon) \underline{G}_{\alpha^{\prime}}^{d c}(\varepsilon+\omega),
$$

where a summation over $c e$ spin has led to the tensor

$$
\theta_{\gamma \gamma^{\prime}}=\frac{1}{2} \sum_{\sigma, \sigma^{\prime}} \tau_{\sigma \sigma^{\prime}}^{i} \tau_{\gamma \gamma^{\prime}}^{i} \tau_{\sigma^{\prime} \sigma}^{j} \tau_{\gamma^{\prime} \gamma}^{j}=\delta_{\gamma \gamma^{\prime}}+2 \tau_{\gamma \gamma^{\prime}}^{1}
$$

Contracting the Keldysh indices $c$ and $d$, using Eq. (23), and using the analytical properties of $\mathcal{I}$, the $K$ component of this self-energy may be written as

$$
\begin{aligned}
\sum_{\gamma \lambda}^{K(2)}(\omega)= & \frac{\theta_{\gamma \gamma^{\prime}}}{16 i} J_{\alpha \alpha^{\prime}}^{2} \int \frac{d \varepsilon}{2 \pi}\left[\begin{array}{l}
\alpha \\
\gamma^{\prime}
\end{array} \mathcal{I}^{<}(\varepsilon) G_{\alpha^{\prime}}^{>}(\varepsilon+\omega)\right. \\
& \left.+{ }_{\gamma^{\prime}}^{\alpha} \mathcal{I}^{>}(\varepsilon) G_{\alpha^{\prime}}^{<}(\varepsilon+\omega)\right] .
\end{aligned}
$$

Similarly, from the retarded and advanced components of the self-energy, the imaginary part is found to be

$$
\begin{aligned}
\Gamma_{\gamma \lambda}^{(2)}(\omega)= & \frac{\theta_{\gamma \gamma^{\prime}}}{16} J_{\alpha \alpha^{\prime}}^{2} \int \frac{d \varepsilon}{2 \pi}\left[\begin{array}{l}
\alpha \\
\gamma^{\prime}
\end{array} \mathcal{I}^{<}(\varepsilon) G_{\alpha^{\prime}}^{>}(\varepsilon+\omega)\right. \\
& \left.-{ }_{\gamma^{\prime}}^{\alpha} \mathcal{I}^{>}(\varepsilon) G_{\alpha^{\prime}}^{<}(\varepsilon+\omega)\right],
\end{aligned}
$$

and the lesser component

$$
\Sigma_{\gamma \lambda}^{<(2)}(\omega)=\frac{\theta_{\gamma \gamma^{\prime}}}{16 i} J_{\alpha \alpha^{\prime}}^{2} \int \frac{d \varepsilon_{\alpha}}{2 \pi} \gamma^{\prime} \mathcal{I}^{>}(\varepsilon) G_{\alpha^{\prime}}^{<}(\varepsilon+\omega) .
$$

Using the identities (30) and (31) it is now straightforward to carry out the integral over $\varepsilon$, together with the summation over $\alpha, \alpha^{\prime}$, and $\gamma^{\prime}$, to find that

$$
\Gamma_{\uparrow}^{(2)}(-B / 2)=\frac{\pi}{4}\left[N_{\uparrow \uparrow}^{(2)}+N_{\uparrow \downarrow}^{(2)}+R^{(2)}\right]
$$

and

$$
\Sigma_{\uparrow \lambda}^{<(2)}(-B / 2)=\frac{i \pi}{4}\left[N_{\uparrow \uparrow}^{(2)} n_{\uparrow, \lambda}+N_{\uparrow \downarrow}^{(2)} n_{\downarrow, \lambda}\right],
$$

where the coefficients are given by

$$
\begin{gathered}
N_{\uparrow \uparrow}^{(2)}=g_{L R}^{2} V \operatorname{coth}(V / 2 T)+\left(g_{L L}^{2}+g_{R R}^{2}\right) T \\
N_{\uparrow \downarrow}^{(2)}=2\left(g_{L L}^{2}+g_{R R}^{2}\right) B[1+N(B)]+2 g_{L R}^{2}[(B+V)\{1+N(B \\
+V)\}+(B-V)\{1+N(B-V)\}] \\
R^{(2)}=-2\left(g_{L L}^{2}+g_{R R}^{2}+2 g_{L R}^{2}\right) B
\end{gathered}
$$

and $N(\omega)=1 /\left(e^{\omega / T}-1\right)$ is the Bose function. While the coefficients $N_{\gamma \gamma^{\prime}}$ do not depend on $\lambda, R^{(2)}$ is in fact given by $\left(n_{\downarrow, \lambda}-1\right) 8 g^{2} B$. Since, however, the factor $n_{\downarrow, \lambda}$ vanishes in the limit of $\lambda \rightarrow \infty$ and leaves behind a term which remains finite, we can simply omit this term and employ the identity (41) for $R$.

\section{Third-order self-energy}

The third-order self-energy diagram depicted in Fig. 3 gives rise to two different terms, corresponding to two different orientations on the $c e$ loop. One involves two Cooper bubbles and the other, two Peierls bubbles, and the combined $a b$ component translates to

$$
\begin{aligned}
\underline{\Sigma}_{\gamma \lambda}^{a b(3)}(\omega)= & \frac{i}{64} J_{\alpha \alpha^{\prime}} J_{\alpha^{\prime} \alpha^{\prime \prime}} J_{\alpha^{\prime \prime} \alpha} \int \frac{d \varepsilon}{2 \pi}\left[\theta_{\gamma \gamma^{\prime} \gamma^{\prime \prime}}^{P} \Lambda_{a b^{\prime}}^{c^{\prime} d}\right. \\
& \times{ }_{\gamma^{\prime \prime}}^{\alpha} \underline{\mathcal{K}}_{b^{\prime} a^{\prime \prime}}^{d^{\prime \prime} c^{\prime}}(\varepsilon) \Lambda_{a^{\prime \prime} b^{\prime \prime} \gamma^{\prime}}^{c^{\prime \prime} d^{\prime \prime} \alpha_{b^{\prime} a^{\prime}}} \underline{\mathcal{K}}_{b^{\prime} c^{\prime \prime}}(\varepsilon) \Lambda_{a^{\prime} b}^{c d^{\prime} b} \\
& \times \underline{G}_{\alpha^{\prime \prime}}^{d c}(\varepsilon+\omega)+\theta_{\gamma \gamma^{\prime} \gamma^{\prime \prime}}^{C} \Lambda_{a b^{\prime} \gamma^{\prime \prime}}^{c d^{\prime} \alpha} \underline{\mathcal{K}}_{b^{\prime} a^{\prime \prime}}^{d^{\prime} c^{\prime \prime}}(\varepsilon) \\
& \left.\times \Lambda_{a^{\prime \prime} b^{\prime \prime} \gamma^{\prime}}^{c^{\prime \prime} d^{\prime \prime} \alpha^{\prime}} \underline{\mathcal{K}}_{b^{\prime \prime} a^{\prime}}^{d^{\prime \prime} c^{\prime}}(\varepsilon) \Lambda_{a^{\prime} b}^{c^{\prime} d} \underline{G}_{\alpha^{\prime \prime}}^{d c}(\varepsilon-\omega)\right],
\end{aligned}
$$

where a summation over $c e$ spin has produced the tensor

$$
\begin{aligned}
\theta_{\gamma \gamma^{\prime} \gamma^{\prime \prime}}^{P} & =-2 i \epsilon_{i j k} \tau_{\gamma \gamma^{\prime}}^{i} \tau_{\gamma^{\prime} \gamma^{\prime \prime}}^{j} \tau_{\gamma^{\prime \prime} \gamma}^{k} \\
& =4\left[\delta_{\gamma \gamma^{\prime}} \tau_{\gamma^{\prime} \gamma^{\prime \prime}}^{1}+\delta_{\gamma \gamma^{\prime \prime}} \tau_{\gamma \gamma^{\prime}}^{1}+\delta_{\gamma^{\prime} \gamma^{\prime \prime}} \tau_{\gamma \gamma^{\prime}}^{1}\right]
\end{aligned}
$$


and $\theta_{\gamma \gamma^{\prime} \gamma^{\prime \prime}}^{C}=-\theta_{\gamma \gamma^{\prime} \gamma^{\prime \prime}}^{P}$. A contraction of the relevant Keldysh indices allows one to express Eq. (42) as a sum of products of two vertex functions. Using again the relations derived in Sec. II A, together with the analytical properties of $\mathcal{I}$, the $K$ component of the third-order self-energy may be written as

$$
\begin{aligned}
\sum_{\gamma \lambda}^{K(3)}(\omega)= & \frac{1}{128 i} \theta_{\gamma \gamma^{\prime} \gamma^{\prime \prime}}^{P} J_{\alpha \alpha^{\prime} \alpha^{\prime \prime}}^{3} \int \frac{d \varepsilon}{2 \pi} \operatorname{Re}\left[_{\gamma^{\prime \prime}}^{\alpha} \mathcal{I}(\varepsilon)\right] \\
& \times\left[{ }_{\gamma^{\prime}}^{\alpha^{\prime}} \mathcal{I}^{<}(\varepsilon) G_{\alpha^{\prime \prime}}^{>}(\varepsilon+\omega)\right. \\
& \left.+{ }_{\gamma^{\prime}}^{\alpha^{\prime}} \mathcal{I}^{>}(\varepsilon) G_{\alpha^{\prime \prime}}^{<}(\varepsilon+\omega)\right]+(V \leftrightarrow-V),
\end{aligned}
$$

where we have introduced the shorthand $J_{\alpha \alpha^{\prime} \alpha^{\prime \prime}}^{3}$ $=J_{\alpha \alpha^{\prime}} J_{\alpha^{\prime} \alpha^{\prime \prime}} J_{\alpha^{\prime \prime} \alpha}$. The imaginary part takes the similar form

$$
\begin{aligned}
\Gamma_{\gamma \lambda}^{(3)}(\omega)= & \frac{1}{128} \theta_{\gamma \gamma^{\prime} \gamma^{\prime \prime}}^{P} J_{\alpha \alpha^{\prime} \alpha^{\prime \prime}}^{3} \int \frac{d \varepsilon}{2 \pi} \operatorname{Re}\left[_{\gamma^{\prime \prime}}^{\alpha} \mathcal{I}(\varepsilon)\right] \\
& \times\left[{ }_{\gamma^{\prime}}^{\alpha^{\prime}} \mathcal{I}^{<}(\varepsilon) G_{\alpha^{\prime \prime}}^{>}(\varepsilon+\omega)\right. \\
& \left.-{ }_{\gamma^{\prime}}^{\alpha^{\prime}} \mathcal{I}{ }^{>}(\varepsilon) G_{\alpha^{\prime \prime}}^{<}(\varepsilon+\omega)\right]+(V \leftrightarrow-V),
\end{aligned}
$$

and the lesser component reads

$$
\begin{aligned}
& \Sigma_{\gamma \lambda}^{<(3)}(\omega)=\frac{1}{128 i} \theta_{\gamma \gamma^{\prime} \gamma^{\prime \prime}}^{P} J_{\alpha \alpha^{\prime} \alpha^{\prime \prime}}^{3} \int \frac{d \varepsilon}{2 \pi} \operatorname{Re}\left[_{\gamma^{\prime \prime}}^{\alpha} \mathcal{I}(\varepsilon)\right] \\
& \times_{\gamma^{\prime}}^{\alpha^{\prime}} \mathcal{I}^{>}(\varepsilon) G_{\alpha^{\prime \prime}}^{<}(\varepsilon+\omega)+(V \leftrightarrow-V) .
\end{aligned}
$$

Comparing to the second-order result, the basic difference is the presence of the extra real part of the vertex function, which provides the logarithmic enhancement underlying the Kondo effect.

Again, the relevant integrations and summations are carried out using the specific form of the various components of $\mathcal{I}$, and the result is expressed just as in Eqs. (37) and (38), but with new third-order coefficients

$$
\begin{aligned}
N_{\uparrow \uparrow}^{(3)}= & 2 g_{L R}^{2} g_{d}\left\{\left[T+(V+B) \operatorname{coth}\left(\frac{V}{2 T}\right)\right] \ln \frac{D}{|V+B|}\right. \\
& \left.+\left[T+(V-B) \operatorname{coth}\left(\frac{V}{2 T}\right)\right] \ln \frac{D}{|V-B|}\right\} \\
& +2\left(g_{L L}^{3}+g_{R R}^{3}\right) T \ln \frac{D}{|B|}, \\
N_{\uparrow \downarrow}^{(3)}= & 4 g_{L R}^{2} g_{d}\left\{[ 1 + N ( B + V ) ] \left[(B+V) \ln \frac{D}{|B+V|}+B \ln \frac{D}{|B|}\right.\right. \\
& \left.+V \ln \frac{D}{|V|}\right]+[1+N(B-V)]\left[(B-V) \ln \frac{D}{|B+V|}\right. \\
& \left.+B \ln \frac{D}{|B|}-V \ln \frac{D}{|V|}\right]+[1+N(B)]\left[(B+V) \ln \frac{D}{|B+V|}\right. \\
& \left.\left.+(B-V) \ln \frac{D}{|B-V|}\right]\right\}+4\left(g_{L L}^{3}+g_{R R}^{3}\right)
\end{aligned}
$$




$$
n_{\gamma \lambda}(\omega)=\left[1-\Sigma_{\gamma \lambda}^{>}(\omega) / \Sigma_{\gamma \lambda}^{<}(\omega)\right]^{-1} .
$$

In this equation the self-energies $\Sigma_{\gamma \lambda}^{<,>}$are determined in perturbation theory as integrals involving the unknown distribution function $n_{\gamma \lambda}(\omega)$. However, in bare perturbation theory, where there is no dressing of internal lines, $n_{\gamma \lambda}(\omega)$ is always multiplied by the unrenormalized $p f$ spectral function (12) inside the integrals, and only the occupation numbers $n_{\gamma \lambda} \equiv n_{\gamma \lambda}(-\gamma B / 2)$ will appear in $\Sigma^{<,>}$. In our case, the quantum Boltzmann equation can thus be solved without any feedback from the retarded and advanced Dyson equations, and we can focus our attention on the on-shell occupation numbers $n_{\gamma \lambda}$.

Dividing out the spectral function and setting $\omega=-\gamma B / 2$, Eq. (48) takes the form

$$
i \Gamma_{\gamma \lambda}(-\gamma B / 2) n_{\gamma \lambda}=\Sigma_{\gamma \lambda}^{<}(-\gamma B / 2) .
$$

Since $n_{\gamma \lambda}$, and thereby $\Sigma_{\gamma \lambda}^{<}$vanishes in the limit of $\lambda \rightarrow \infty$, the whole equation may be divided by $\langle Q\rangle_{\lambda}$ and after this limit has been taken one can establish the same equation for the physical, projected, occupation numbers. Expressed in terms of the coefficients defined in Eqs. (37) and (38), we arrive at the equation

$$
\left(N_{\uparrow \uparrow}+N_{\uparrow \downarrow}+R\right) n_{\uparrow}=N_{\uparrow \uparrow} n_{\uparrow}+N_{\uparrow \downarrow} n_{\downarrow},
$$

which can be viewed as a rate equation with the transition rates $W_{\downarrow \uparrow}=(\pi / 4)\left(N_{\uparrow \downarrow}+R\right)$ and $W_{\uparrow \downarrow}=(\pi / 4) N_{\uparrow \downarrow}$. This equation is readily solved together with the constraint equation $n_{\uparrow}+n_{\downarrow}=1$ and one finds that

$$
n_{\uparrow}=\frac{N_{\uparrow \downarrow}}{2 N_{\uparrow \downarrow}+R},
$$

or expressed in terms of the magnetization $M=n_{\uparrow}-n_{\downarrow}$,

$$
M=\frac{-R}{2 N_{\uparrow \downarrow}+R} .
$$

Note that to obtain the observable magnetization to order $g^{2} \ln (D)$, it is not sufficient to consider only the on-shell occupations appearing in Eqs. (53)-(55). In addition, one has to consider also contributions from the $p f$ spectral function [see Eq. (14)], which are discussed in detail in the Appendix.

Inserting the second-order expressions (39)-(41), one obtains

$$
M(B, V)=\frac{\left(g_{L L}^{2}+g_{R R}^{2}+2 g_{L R}^{2}\right) B}{\left(g_{L L}^{2}+g_{R R}^{2}\right) B \operatorname{coth}\left(\frac{B}{2 T}\right)+g_{L R}^{2}\left[(B+V) \operatorname{coth}\left(\frac{B+V}{2 T}\right)+(B-V) \operatorname{coth}\left(\frac{B-V}{2 T}\right)\right]}
$$

which, up to a factor of 2 in the definition of $M$, is exactly what was found in Eq. (4) of Ref. 55, where a rate equation such as Eq. (53) was solved using second-order transition rates. Including the third-order corrections (44)-(46), we obtain

$$
M=\mathcal{N} / \mathcal{D}
$$

with

$$
\begin{aligned}
\mathcal{N}= & 4 g_{L R}^{2} g_{d}\left[(V+B) \ln \frac{D}{|V+B|}-(V-B) \ln \frac{D}{|V-B|}\right] \\
& +B \sum_{\alpha}\left(g_{L \alpha}^{2}+g_{R \alpha}^{2}\right)\left(1+2 g_{\alpha \alpha} \ln \frac{D}{|B|}\right), \\
\mathcal{D}= & \operatorname{coth}\left(\frac{B}{2 T}\right)\left\{2 g _ { L R } ^ { 2 } g _ { d } \left[(V+B) \ln \frac{D}{|V+B|}\right.\right. \\
& \left.\left.-(V-B) \ln \frac{D}{|V-B|}\right]+B \sum_{\alpha} g_{\alpha \alpha}^{2}\left(1+2 g_{\alpha \alpha} \ln \frac{D}{|B|}\right)\right\} \\
& +\operatorname{coth}\left(\frac{V+B}{2 T}\right) g_{L R}^{2}\left[(V+B)\left(1+2 g_{d} \ln \frac{D}{|V+B|}\right)\right.
\end{aligned}
$$

$$
\begin{aligned}
& \left.+2 V g_{d} \ln \frac{D}{|V|}+2 B g_{d} \ln \frac{D}{|B|}\right]+\operatorname{coth}\left(\frac{V-B}{2 T}\right) g_{L R}^{2}[(V-B) \\
& \left.\times\left(1+2 g_{d} \ln \frac{D}{|V-B|}\right)+2 V g_{d} \ln \frac{D}{|V|}-2 B g_{d} \ln \frac{D}{|B|}\right],
\end{aligned}
$$

using again the shorthand $\ln (D /|x|)=\ln \left(D / \sqrt{x^{2}+T^{2}}\right)$. Both numerator $\mathcal{N}$ and denominator $\mathcal{D}$ of the magnetization $M$ are calculated only to order $g^{2}+g^{3} \ln [\cdots]$, and $M$ should therefore also be expanded. As expected, the Kondo effect reveals itself in logarithmic enhancements, and interestingly enough the logarithmic corrections to $M$ come as $[1+g \ln (\cdots)]$ rather than $\left[1+g^{2} \ln (\cdots)\right]$, which is found in equilibrium. In the limit where $T \gg V$, the logarithmic corrections from $\mathcal{N}$ and $\mathcal{D}$ cancel and we recover the usual thermal magnetization $M=\tanh (B / 2 T)$. In the highly asymmetric case, where $g_{L L} \gg g_{R R}, g_{L R}$, a similar cancellation takes place and one finds again that $M=\tanh (B / 2 T)$. In the Appendix, we demonstrate how the observable magnetization receives additional corrections of order $g^{2} \ln (\cdots)$, arising from self-energy corrections to the $p f$ spectral function. Nevertheless, in the case where $V \gg T$, such additional corrections are subleading.

In Fig. 4 we plot the magnetization and the corresponding susceptibility $\partial M / \partial B$ as functions of $B / V$ for $T \ll V$, while Fig. 5 investigates the $V$ dependence of $M$ and $\partial M / \partial V$, 


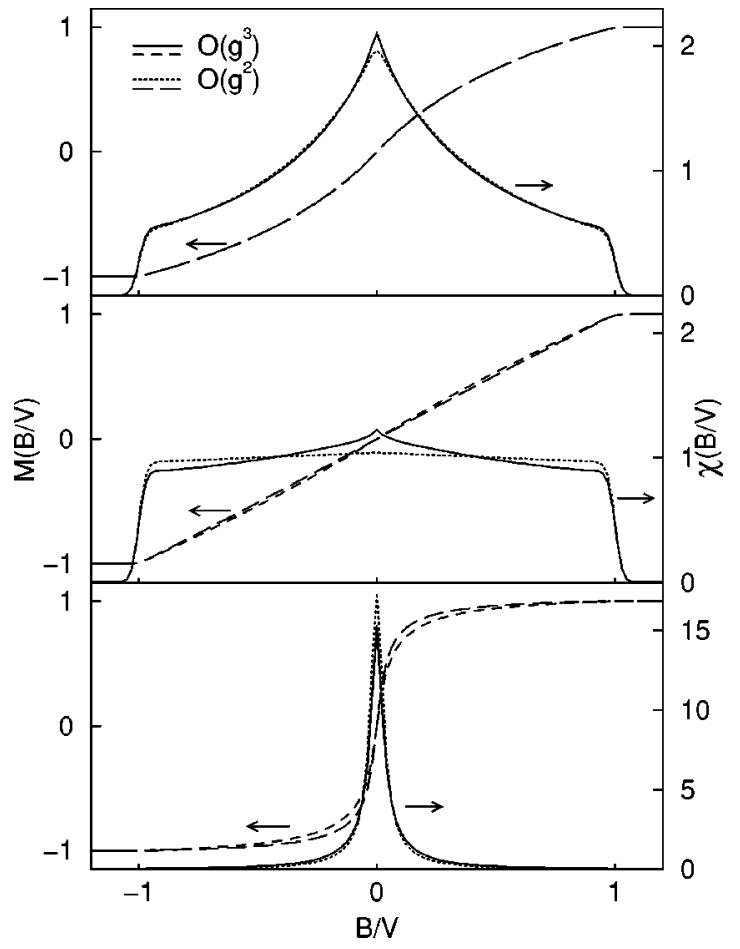

FIG. 4. Nonequilibrium magnetization (dashed and long-dashed lines) and susceptibility (full and dotted lines) as functions of $B / V$, for $T=10^{-2} V$ and $D=10^{3} V$. Solid and dashed (dotted and longdashed) lines correspond to 3.(2.) order perturbation theory. Both quantities have been expanded to first order in the exchange couplings. Upper panel: $g_{L L}=g_{R R}=g_{L R}=0.01$. Middle panel: $g_{L L}$ $=g_{R R}=0.01$ and $g_{L R}=0.05$. Lower panel: $g_{L L}=g_{R R}=0.05$ and $g_{L R}=0.01$ (note the different scale for $\chi$ ). Corrections from third order are more pronounced for $g_{L R}^{2} \neq g_{L L} g_{R R}$.

which will both influence the conductance. Roughly speaking, the magnetization curve resembles the usual thermal magnetization with $V$ replacing $T$, and the impurity-spin becomes polarized only when $B$ exceeds $V$. However, structures close to $V \sim B$ are much sharper and obtain completely different logarithmic corrections compared to the equilibrium case. The exchange-correlations change the slopes of the magnetization, which is clearly seen in the susceptibility (Fig. 4) and in $\partial M / \partial V$ (Fig. 5) which become sharper spiked when including third-order perturbations. In the limit of $V$ $\gg\left[\left(g_{L L}^{2}+g_{R R}^{2}\right) / 2 g_{L R}^{2}\right] B, V \gg B$, and small $T$, one may expand to find

$$
\begin{aligned}
M \approx & \frac{B}{V}\left\{\frac{g_{L L}^{2}+g_{R R}^{2}+2 g_{L R}^{2}}{2 g_{L R}^{2}}+\frac{\left(g_{L L}+g_{R R}\right)\left(g_{L L}^{2}+g_{R R}^{2}\right)}{g_{L R}^{2}}\right. \\
& \left.\times\left[\left(1+\frac{g_{L R}^{2}-g_{L L} g_{R R}}{g_{L L}^{2}+g_{R R}^{2}}\right) \ln \frac{D}{B}-\ln \frac{D}{V}\right]\right\},
\end{aligned}
$$

which simplifies to $M=(2 B / V)(1+2 g \ln |V / B|)$ when $g_{L L}$ $=g_{R R}=g_{L R}$.

In the regime where the bias voltage $V$ does not supply sufficient energy to flip the spin, $V<B$ and $B-V \gg T$, the magnetization is at its equilibrium value $M=1$ to first order

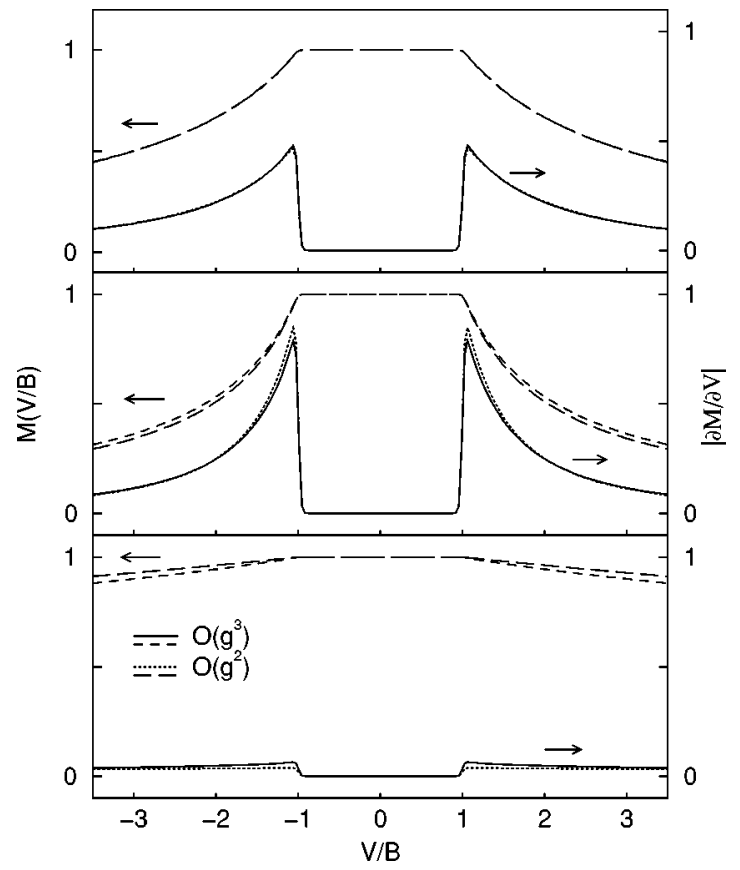

FIG. 5. Nonequilibrium magnetization as a function of $V / B$, for $T=10^{-2} B$ and $D=10^{3} B$. The two lower curves in each panel show the absolute value of the slope, i.e., $|\partial M / \partial V|$. This quantity enters the conductance shown in Fig. 7. Solid (dotted) lines correspond to 3.(2.) order perturbation theory. Both quantities have been expanded to first order in the exchange couplings. Upper panel: $g_{L L}$ $=g_{R R}=g_{L R}=0.01$. Middle panel: $g_{L L}=g_{R R}=0.01$ and $g_{L R}$ $=0.05$. Lower panel: $g_{L L}=g_{R R}=0.05$ and $g_{L R}=0.01$.

in $g$. However, as soon as $V>B$, voltage-induced spin flips are possible and the magnetization is reduced (see Fig. 5). For $V>B$ and $T \ll V-B \ll B$ we obtain

$$
\begin{aligned}
M \approx & 1-\left(\frac{V-B}{B}\right) \frac{2 g_{L R}^{2}}{g_{L L}^{2}+g_{R R}^{2}+2 g_{L R}^{2}}\left\{1+\left(g_{L L}+g_{R R}\right)\left[\ln \frac{D}{V-B}\right.\right. \\
& \left.\left.-\left(1+2 \frac{g_{L R}^{2}-g_{L L} g_{R R}}{g_{L L}^{2}+g_{R R}^{2}+2 g_{L R}^{2}}\right) \ln \frac{D}{B}\right]\right\} .
\end{aligned}
$$

It is interesting to note that the structure of logarithmic corrections is rather special in the case when the Hamiltonian (1) is derived from an underlying Anderson model by means of a Schrieffer-Wolf transformation. In this case the exchange couplings are related as $g_{L R}^{2}=g_{L L} g_{R R}$ (cf., e.g., Ref. 41 ) and for such realizations of the model all leading $\ln D$ contributions cancel while logarithmic corrections of the form $\ln (V / B)$ or $\ln (B /(V-B))$ remain. For example, Eq. (60) simplifies to

$$
M \approx \frac{B}{V}\left\{1+\frac{g_{L L}^{2}+g_{R R}^{2}}{2 g_{L L} g_{R R}}\left[1+4 g_{d} \ln \frac{V}{B}\right]\right\},
$$

and Eq. (61) takes the form

$$
M \approx 1-\frac{V-B}{B} \frac{2 g_{L L} g_{R R}}{\left(g_{L L}+g_{R R}\right)^{2}}\left[1+2 g_{d} \ln \frac{B}{V-B}\right]
$$


This explains why the plots of the magnetization in the upper panels in Figs. 4 and 5 hardly differ from second to third order, while more pronounced effects are seen in the lower panels where $g_{L R}^{2} \neq g_{L L} g_{R R}$.

From Eq. (62) we see that the peak at $B=0$ in the magnetic susceptibility, seen in Fig. 4, grows as $\ln (|V| / T)$ for $T$ $\ll V$. Also, from Eq. (60) we learn that the high-voltage tails in Fig. 5 fall off as $1 / V$. The derivative $\partial M / \partial V$, shown in
Fig. 5, displays a peak near $V=B$ already in second-order perturbation theory which is then slightly enhanced or reduced by the third-order correction, depending on the relative size of $g_{d}$ and $g_{L R}$. As we shall see in Sec. IV, these features turn out to have a marked influence on the conductance.

For arbitrary $T$ and $V$, the susceptibility at $B=0$ takes the following form:

$$
\chi=\frac{2 g_{L R}^{2}\left(1+2 g_{d} \ln \frac{D}{T}+2 g_{d} \ln \frac{D}{|V|}\right)+\sum_{\alpha} g_{\alpha \alpha}\left(g_{\alpha \alpha}+2 g_{\alpha \alpha}^{2} \ln \frac{D}{T}+2 g_{L R}^{2} \ln \frac{D}{|V|}\right)}{2 V \operatorname{coth}\left(\frac{V}{2 T}\right) g_{L R}^{2}\left(1+4 g_{d} \ln \frac{D}{|V|}\right)+2 T \sum_{\alpha} g_{\alpha \alpha}\left(g_{\alpha \alpha}+2 g_{\alpha \alpha}^{2} \ln \frac{D}{T}+2 g_{L R}^{2} \ln \frac{D}{|V|}\right)} .
$$

In the limit of $T \gg V$ the logarithms all take the form $\ln (D / T)$; the corrections in numerator and denominator cancel, and we are left with the usual Curie law $\chi=1 /(2 T)$. In the extreme nonequilibrium situation, however, where $V \gg T$, the corrections no longer cancel and we are left with a complicated fraction times $1 / V$.

It is important to note that numerator and denominator in Eq. (64) have a rather different structure of logarithmic corrections, e.g., the $g_{L R}^{2}$ term in the numerator receives corrections of the form $2 g_{d}[\ln (D / T)+\ln (D /|V|)]$ whereas the corresponding term in the denominator has the form $4 g_{d} \ln (D /|V|)$. This observation was the basis of our claim in Ref. 45 that the perturbative renormalization group has to be formulated in terms of coupling functions which depend on the energy of the incoming electron. This will be explained in more detail in a forthcoming publication. ${ }^{50}$

\section{NONLINEAR TUNNELING CURRENT}

The current operator measuring the charge flow from left to right lead is found from the equation of motion for the charge density in the left lead:

$$
\partial_{t} n_{L}=i\left[H, n_{L}\right]
$$

The expectation value at time $t$ is

$$
j_{L}=i J_{L R} \sum_{\mathbf{k}, \mathbf{k}^{\prime}}\left\langle\vec{S}(t) \cdot\left[c_{L \mathbf{k}^{\prime} \sigma^{\prime}}^{\dagger}(t) \vec{\tau}_{\sigma^{\prime} \sigma c_{R \mathbf{k} \sigma}}(t)-L \leftrightarrow R\right]\right\rangle,
$$

and by defining a two-particle Keldysh contourordered correlation function

$$
\begin{aligned}
& D_{L R}\left(\tau, \tau^{\prime}\right) \\
& \quad=(-i)^{2} \sum_{\mathbf{k}, \mathbf{k}^{\prime}}\left\langle T_{c_{K}}\left\{c_{L \mathbf{k}^{\prime} \sigma^{\prime}}^{\dagger}(\tau) \frac{\vec{\tau}_{\sigma^{\prime} \sigma}}{2} c_{R \mathbf{k} \sigma}(\tau) \cdot \vec{S}\left(\tau^{\prime}\right)\right\}\right),
\end{aligned}
$$

the current may be expressed simply by the imaginary part of the Keldysh component

$$
j_{L}=J_{L R} \operatorname{Im}\left[D_{L R}^{K}(t, t)\right] .
$$

The lowest-order contribution to this correlation function is obtained from the first Feynman diagram in Fig. 6, which translates to

$$
\begin{aligned}
D_{L R}^{K(1)}(t, t)= & -\frac{\theta_{\gamma \gamma^{\prime}}}{8 i} J_{L R} \int \frac{d \varepsilon}{2 \pi} \int \frac{d \omega}{2 \pi} \int \frac{d \Omega}{2 \pi} \\
& \times \widetilde{\gamma}_{a^{\prime} b^{\prime}}^{1} \mathcal{G}_{\gamma^{\prime}}^{b a^{\prime}}(\omega) \mathcal{G}_{\gamma}^{b^{\prime} a}(\omega+\Omega) \\
& \times \Lambda_{a b}^{c d} G_{R}^{d^{\prime} c}(\varepsilon) G_{L}^{d c^{\prime}}(\varepsilon+\Omega) \gamma_{c^{\prime} d^{\prime}}^{2},
\end{aligned}
$$

with measuring vertices $\tilde{\gamma}^{1}$ and $\gamma^{2}$ defined in Eqs. (10) and (11) and the spin tensor from Eq. (33). Contracting the Keldysh indices and expressing things in terms of the vertex function $\mathcal{I}$, this simplifies to

$$
\begin{aligned}
D_{L R}^{K(1)}(t, t)= & \frac{\theta_{\gamma \gamma^{\prime}}}{128 i} J_{L R} \int \frac{d \varepsilon}{2 \pi}\left[{ }_{\gamma}^{L} \mathcal{I}^{K}(\varepsilon){ }_{\gamma^{\prime}}^{R} \mathcal{I}^{R}(\varepsilon)\right. \\
& \left.+{ }_{\gamma}^{L} \mathcal{I}^{A}(\varepsilon){ }_{\gamma^{\prime}}^{R} \mathcal{I}^{K}(\varepsilon)\right],
\end{aligned}
$$

which in turn leads to

$$
j_{L}^{(2)}=\frac{\theta_{\gamma \gamma^{\prime}}}{32 i} J_{L R}^{2} \int \frac{d \varepsilon}{2 \pi} \operatorname{Im}\left[{ }_{\gamma}^{R} \mathcal{I}(\varepsilon)\right]_{\gamma^{\prime}}^{L} \mathcal{I}^{K}(\varepsilon)-(V \leftrightarrow-V) .
$$

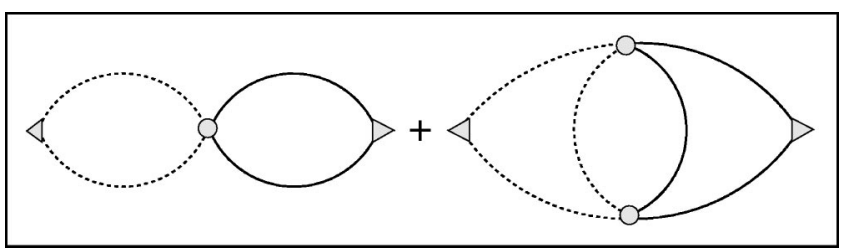

FIG. 6. Unlabeled Feynman diagrams for $D_{L R}$. Triangles denote the bare measurement vertices. 
Evaluating this expression and performing the projection, one finds the physical charge current to be

$$
\begin{aligned}
j_{L}^{(2)}= & \frac{\pi}{4} g_{L R}^{2}\left\{3 V-M\left[(V+B) \operatorname{coth}\left(\frac{V+B}{2 T}\right)\right.\right. \\
& \left.\left.-(V-B) \operatorname{coth}\left(\frac{V-B}{2 T}\right)\right]\right\} .
\end{aligned}
$$

The magnetization entering this formula is the on-shell magnetization determined in Sec. III B, and since we include no dressing of internal lines this quantity receives no additional renormalization from the retarded and advanced Dyson equations.

The second-order corrections to the correlation function $D_{L R}$ are contained in the second diagram in Fig. 6. After contracting indices with the measurement vertices, this may be written in terms of Peierls and Cooper bubbles as

$$
\begin{aligned}
D_{L R}^{K(2)}(t, t)= & -\frac{1}{128} J_{L \alpha} J_{\alpha R} \theta_{\gamma \gamma^{\prime} \gamma^{\prime \prime}}^{P} \int \frac{d \varepsilon}{2 \pi}\left[\Lambda_{a^{\prime \prime} b \gamma^{\prime}}^{c d^{\prime \prime} R} \underline{\mathcal{K}}_{b a^{\prime}}^{d^{\prime} c}(\varepsilon)\right. \\
& \times \Lambda_{a b^{\prime \prime} \gamma^{\prime \prime}}^{c^{\prime \prime} d \alpha} \underline{\mathcal{K}}_{b^{\prime \prime} a^{\prime \prime}}^{d^{\prime \prime} c^{\prime \prime}}(\varepsilon){ }_{\gamma}^{L} \underline{\mathcal{K}}_{a^{\prime} a}^{d} \bar{d}^{\prime} \\
& -\Lambda_{a^{\prime \prime} b \gamma^{\prime}{ }^{\prime \prime}}^{c^{\prime \prime} d L} \overline{\mathcal{K}}_{b a^{\prime}}^{d c^{\prime}}(\varepsilon) \Lambda_{a b^{\prime \prime}}^{c d^{\prime \prime}} \\
& \left.\times{ }_{\gamma^{\prime \prime}}^{\alpha} \underline{\mathcal{K}}_{b^{\prime \prime} a^{\prime \prime}}^{d^{\prime \prime} c^{\prime \prime}}(\varepsilon)_{\gamma}^{R} \underline{\mathcal{K}}_{\bar{a}^{\prime} a}^{\bar{c}^{\prime} c}(\varepsilon)\right]
\end{aligned}
$$

which upon full contraction leads to

$$
\begin{aligned}
D_{L R}^{K(2)}(t, t)= & \frac{1}{512 i} J_{L \alpha} J_{\alpha R} \theta_{\gamma \gamma^{\prime} \gamma^{\prime \prime}}^{P} \\
& \times \int \frac{d \varepsilon}{2 \pi}\left[{ }_{\gamma^{\prime}}^{R} \mathcal{I}^{K}(\varepsilon){ }_{\gamma^{\prime \prime}}^{\alpha} \mathcal{I}^{A}(\varepsilon){ }_{\gamma}^{L} \mathcal{I}^{A}(\varepsilon)\right. \\
& +{ }_{\gamma^{\prime}}^{R} \mathcal{I}^{R}(\varepsilon){ }_{\gamma^{\prime \prime}}^{\alpha} \mathcal{I}^{K}(\varepsilon){ }_{\gamma}^{L} \mathcal{I}^{A}(\varepsilon) \\
& \left.+{ }_{\gamma^{\prime}}^{R} \mathcal{I}^{R}(\varepsilon){ }_{\gamma^{\prime \prime}}^{\alpha} \mathcal{I}^{R}(\varepsilon){ }_{\gamma^{L}} \mathcal{I}^{K}(\varepsilon)\right]+(V \leftrightarrow-V) .
\end{aligned}
$$

Inserting this into Eq. (67), we end up with

$$
\begin{aligned}
j_{L}^{(3)}= & \frac{\theta_{\gamma \gamma^{\prime} \gamma^{\prime \prime}}^{P}}{64 i} J_{L R}^{2}\left(J_{L L}+J_{R R}\right) \int \frac{d \varepsilon}{2 \pi} \operatorname{Re}\left[{ }_{\gamma}^{L} \mathcal{I}(\varepsilon)+{ }_{\gamma}^{R} \mathcal{I}(\varepsilon)\right] \\
& \times \operatorname{Im}\left[{ }_{\gamma^{\prime \prime}}^{R} \mathcal{I}(\varepsilon)\right]_{\gamma^{\prime}}^{L} \mathcal{I}^{K}(\varepsilon)-(V \leftrightarrow-V),
\end{aligned}
$$

which may finally be evaluated and projected to obtain the physical current

$$
\begin{aligned}
j_{L}= & \frac{\pi}{4} g_{L R}^{2}\left\{V\left(1+4 g_{d} \ln \frac{D}{|V|}\right)+(V+B)\left(1+4 g_{d} \ln \frac{D}{|V+B|}\right)\right. \\
& +(V-B)\left(1+4 g_{d} \ln \frac{D}{|V-B|}\right)-M\left[\operatorname{coth}\left(\frac{V+B}{2 T}\right)\right. \\
& \times\left\{(V+B)\left(1+2 g_{d} \ln \frac{D}{|V+B|}\right)+2 V g_{d} \ln \frac{D}{|V|}\right.
\end{aligned}
$$

$$
\begin{aligned}
& \left.+2 B g_{d} \ln \frac{D}{|B|}\right\}-\operatorname{coth}\left(\frac{V-B}{2 T}\right)\{(V-B) \\
& \left.\left.\left.\times\left(1+2 g_{d} \ln \frac{D}{|V-B|}\right)+2 V g_{d} \ln \frac{D}{|V|}-2 B g_{d} \ln \frac{D}{|B|}\right\}\right]\right\} .
\end{aligned}
$$

Here again, $\ln (D /|x|)$ is shorthand for $\ln \left(D / \sqrt{x^{2}+T^{2}}\right)$ as all logarithms are cut off by $T$. The current has acquired logarithmic corrections, which again enter as $[1+g \ln (\cdots)]$, and in zero magnetic field we recover the conductance obtained earlier in Refs. 21 and 41 (reinstalling $e$ and $\hbar$ ):

$$
G(V)=\frac{e^{2}}{\pi \hbar}\left(\frac{\pi}{2} g_{L R}\right)^{2} 3\left(1+4 g_{d} \ln \frac{D}{|e V|}\right) .
$$

In a finite magnetic field, however, the proliferation of logarithms is more intricate. While the logarithmic structure in Eq. (75) was derived already by Appelbaum, ${ }^{21,22}$ he tacitly employed the equilibrium magnetization $M=\tanh (B / 2 T)$, instead of the correct expression (57), and thereby left out a number of logarithmic corrections to the resulting conductance. These early results by Appelbaum are therefore only valid for a local moment coupled dominantly to one electrode, in which case $g_{L L} \gg g_{R R}, g_{L R}$ and therefore indeed $M$ $=\tanh (B / 2 T)$, as was pointed out in Sec. III B. Having solved the quantum Boltzmann equation in Sec. III B, including the leading logarithmic corrections, we can now correct for this omission.

Close to $V=B$, the conductance shows a characteristic jump (see Fig. 7). For $V<B$ and $T \ll B-V \ll V$ we obtain

$$
G(B, V) \approx \frac{e^{2}}{\pi \hbar}\left(\frac{\pi}{2} g_{L R}\right)^{2}\left\{1+2 g_{d}\left[\ln \frac{D}{B}+\ln \frac{D}{B-V}\right]\right\},
$$

while for $V>B$ and $T \ll V-B \ll B$ we get

$$
\begin{aligned}
G(B, V) \approx & \frac{e^{2}}{\pi \hbar}\left(\frac{\pi}{2} g_{L R}\right)^{2}\left\{3+\frac{g_{L R}^{2}}{g^{2}}+2 g_{d}\left[\left(3+\frac{g_{L R}^{2} g_{d}^{2}}{g^{4}}\right) \ln \frac{D}{B}\right.\right. \\
& \left.\left.+\left(3+\frac{g_{L R}^{2}}{g^{2}}\right) \ln \frac{D}{V-B}\right]\right\} .
\end{aligned}
$$

The jump in the conductance is broadened by the temperature $T$ and we obtain for $V, B \gg T \gg|V-B|$

$$
\begin{aligned}
G(B=V, T) \approx & \frac{e^{2}}{\pi \hbar}\left(\frac{\pi}{2} g_{L R}\right)^{2}\left\{2+\frac{g_{L R}^{2}}{2 g^{2}}\right. \\
& \left.+2 g_{d}\left[\left(2+\frac{g_{L R}^{2} g_{d}^{2}}{2 g^{4}}\right) \ln \frac{D}{B}+\left(2+\frac{g_{L R}^{2}}{2 g^{2}}\right) \ln \frac{D}{T}\right]\right\},
\end{aligned}
$$

with $g^{2}=\left(g_{L L}^{2}+g_{R R}^{2}+2 g_{L R}^{2}\right) / 4$. All terms in Eqs. (78) and (79) proportional to $g_{L R}^{2} / g^{2}$ originate from $\partial M / \partial V$ and are therefore not present in Appelbaum's result. The logarithmic divergences $\ln D /|V-B|$ and $\ln D / T$ in Eqs. (77)-(79) seem to 


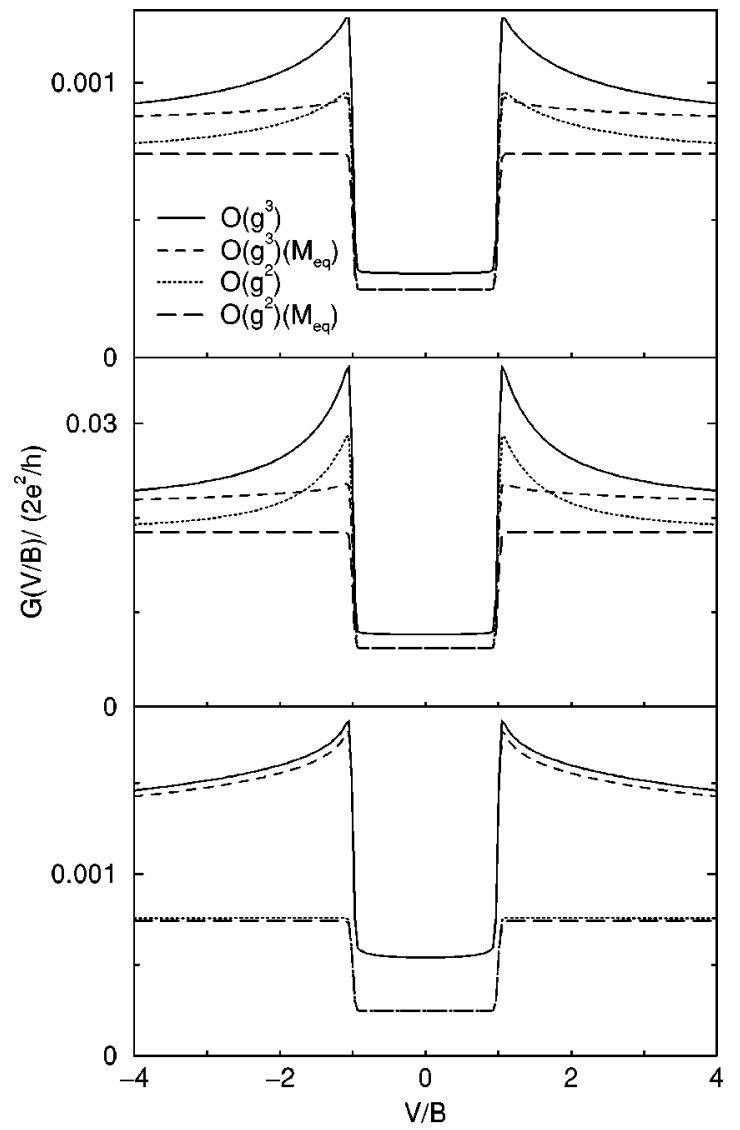

FIG. 7. Conductance, expanded to third order in the exchange couplings, as a function of $V / B$, for $T=10^{-2} B$ and $D=10^{3} B$. Solid and dashed (dotted and long-dashed) lines correspond to 3.(2.) order perturbation theory. Dashed and long-dashed lines have $M=1$ and correspond to Appelbaum's result, which neglects the $V$ dependence of $M$ shown in Fig. 5. Upper panel: $g_{L L}=g_{R R}=g_{L R}=0.01$. Middle panel: $g_{L L}=g_{R R}=0.01$ and $g_{L R}=0.05$. Lower panel: $g_{L L}$ $=g_{R R}=0.05$ and $g_{L R}=0.01$.

suggest that logarithmic corrections may grow large for $V$ $\rightarrow B$ and $T \rightarrow 0$, signaling a transition to the strong-coupling regime, but such behavior will ultimately be prohibited by spin-relaxation processes emanating from the finite current flowing through the dot. As argued in Refs. 56 and 45, the relevant cutoff energy is the voltage dependent spinrelaxation rate $\Gamma \sim g^{2} V$, and for $T,|V-B|<\Gamma$, the diverging logarithms will be replaced by $\ln D / \Gamma$, leading to a finite correction. This cutoff arises as a joint effect of vertex and selfenergy corrections, which will be worked out in detail in a subsequent publication. ${ }^{57}$ The same relaxation rate also broadens the jump in the conductance and Eq. (79) is therefore only valid for $T \gg \Gamma$.

In Fig. 7 we plot the conductance as a function of $V / B$, for $T \ll V$, using both the nonequilibrium magnetization given by Eqs. $(57)-(59)$ and the equilibrium value $(M \approx 1$ since $T \ll B$ ), corresponding to Appelbaum's result. In both cases a cusp develops at $|V| \approx|B|$, beyond which spin-flip tunneling processes are energetically viable. Including the nonequilibrium magnetization, however, leads to a pronounced enhancement of the cusps, coming from $\partial M / \partial V$ (shown in Fig. 5), which is missing in Appelbaum's result. In the lower panel, where $g_{L R}<g_{d}$, Appelbaum's conductance is closer to the true curve, as for small $g_{L R}$ one is closer to equilibrium ( $g_{L R}=0$ is an equilibrium problem).

Our result for the conductance appears to be similar to a plot by Sivan and Wingreen ${ }^{40}$ for the Anderson model, but since that work does not contain an explicit analytical expression for the current and the magnetization it is difficult to compare the two results. Furthermore, logarithmic corrections to the quantum Boltzmann equation appear not to be included in their approach.

In the high-temperature limit $T \gg \max (V, B)$, there is no effect of $V$ on the magnetization, and we recover Appelbaum's result for the current, or rather the result he would have obtained by performing the final energy integrations in his expressions (39) and (60) of Ref. 22. The closed-form expression presented in Ref. 23 is the conductance rather than the current, and as an aside we note that differences from his formulas (where the $T$ dependence arises through hyperbolic tangents) to our Eq. (75) (with hyperbolic cotangents) arise due to an (invalid) approximation by Appelbaum, when he approximated the derivative of the Fermi function by a $\delta$ function. Using the identity $f(\omega+\varepsilon)[1$ $-f(\omega+\Omega)]=N(\varepsilon-\Omega)[f(\omega+\Omega)-f(\omega+\varepsilon)] \quad$ between Fermi and Bose functions, we find $\operatorname{coth}(x)+x \partial_{x} \operatorname{coth}(x)$ instead of $\tanh (x)$ giving rise to a different prefactor $(2 / 3$ instead of 1) for $T$ larger than either $V, B$, or $|V-B|$.

\section{DISCUSSION}

In the present work we have calculated the local magnetization and the charge current through a quantum dot at large bias voltage and in the presence of a magnetic field. We have considered a Kondo model where the dot is represented by a quantum spin $S=1 / 2$, coupled to leads by exchange interaction and tunneling.

Remarkably, the structure of perturbation theory in steady-state nonequilibrium is rather different from that in equilibrium. The main physical reason is that in our problem the occupation on the dot is completely undetermined in the limit of vanishing couplings to the leads and therefore has to be calculated from the solution of the quantum Boltzmann equation (i.e. from a self-consistent Dyson equation). More generally, in equilibrium all distribution functions are given exactly by the "bare" Boltzmann, Fermi, or Bose functions without any corrections from interactions while out of equilibrium these functions have to be calculated and will depend on all the couplings. Probably the most drastic consequence is that for finite voltages the magnetization (56) is modified even for vanishing couplings, i.e., in zeroth order of perturbation theory as has been emphasized in Ref. 56. As a consequence the structure of logarithmic corrections to the magnetization is also rather different out of equilibrium. As the matrix elements of order $g^{2}$ in the quantum Boltzmann equation get corrections of order $g^{3} \ln (D)$, the perturbative magnetization (57) is generically of the form $\left(g^{2}+g^{3}\right.$ $\times \ln (\cdots)) /\left(g^{2}+g^{3} \ln (\cdots)\right) \approx$ const $+O[g \ln (\cdots)]$, to be compared to the equilibrium case where logarithmic corrections arise only to order $g^{2} \ln (\cdots)$.

In this paper, we have calculated the magnetization and 
the current through a Kondo dot to third order in the coupling, including the leading logarithmic terms. In contrast to earlier treatments of this problem, ${ }^{21,22}$ the effect of the nonequilibrium magnetization on the current is incorporated and indeed shown to be important for a typical quantum dot experiment in the Kondo regime. In finite magnetic field the differential conductance exhibits threshold behavior at $V= \pm B$, reflecting the fact that spin-flip tunneling is possible only for $|V|>|B|$. Taking the voltage dependence of the magnetization properly into account, the conductance shows a cusp at $V= \pm B$, already to lowest order in perturbation theory. Going one order higher, logarithmic corrections are found to enhance these cusps even further, increasing the conductance substantially over the threshold plateau, even for magnetic fields much larger than temperature. This behavior clearly calls for a resummation of the perturbation series to infinite order.

The intricate structure of logarithmic corrections, revealed by nonequilibrium perturbation theory, enforces a modified formulation of the perturbative renormalization group. Thinking in terms of poor man's scaling, a resummation of the logarithmic corrections cannot simply be achieved by collecting the logarithmic corrections into a renormalized coupling constant. This is clearly illustrated by Eq. (64) for the susceptibility, which shows that different combinations of logarithms appear in the numerator and in the denominator. However, as we have recently demonstrated, ${ }^{45}$ exactly this structure of leading logarithms is generated in a poor man's scaling approach dealing with energy dependent coupling functions. A detailed account of this approach will be given in a subsequent publication. ${ }^{50}$

An additional important difference between nonequilibrium and equilibrium physics is the fact that in a nonequilibrium situation the current through the system generates substantial noise. As a consequence, quantum coherence is limited to energies above a certain relaxation rate $\Gamma$. The scale $\Gamma$ will therefore cut off all logarithmic divergences which remain in the limit $T \rightarrow 0$ in the perturbative expressions for magnetization and current found in this paper. This important piece of physics is not included in the low-order bare perturbation theory presented here and requires a resummation of subleading self-energy and vertex corrections. This will be demonstrated explicitly in a forthcoming publication. $^{57}$

Historically, the Kondo effect has played an important role in the development of techniques such as the renormalization group ${ }^{4,5}$ to treat strong-coupling problems for systems in thermal equilibrium. It is our hope that the present perturbative calculation of leading logarithmic corrections can serve both as a starting point and a check for future developments of similar methods applicable to systems out of equilibrium.

\section{ACKNOWLEDGMENTS}

We thank P. Coleman, L. Glazman, C. Hooley, J. Kroha, O. Parcollet, and H. Smith for useful discussions. J.P. acknowledges the hospitality of the Ørsted Laboratory at the University of Copenhagen in Denmark, where parts of this work was carried out. This work was supported in part by the CFN and the Emmy Noether program (A.R.) of the DFG.

\section{APPENDIX: MAGNETIZATION AND SELF-ENERGY CORRECTIONS}

In this appendix we calculate the observable magnetization to order $g^{2} \ln (\cdots)$, by including self-energy corrections to the pseudofermion spectral function. Using the prescription (3) for evaluation of a canonical ensemble average, the magnetization is determined as

$$
M=\lim _{\lambda \rightarrow \infty} \frac{n_{\uparrow \lambda}-n_{\downarrow \lambda}}{n_{\uparrow \lambda}+n_{\downarrow \lambda}},
$$

where

$$
n_{\gamma \lambda}=\int_{-\infty}^{\infty} \frac{d \omega}{2 \pi} \mathcal{A}_{\gamma}(\omega) n_{\gamma \lambda}(\omega)
$$

and, in principle, the full frequency dependence of both the spectral and the distribution function is needed to carry out this integral.

To illustrate how such renormalization works out within the pseudofermion approach, we shall commence with the simpler case of thermal equilibrium and consider merely linear response for $B \ll T$. Traditionally, most work regarding the Kondo effect on the magnetic susceptibility in this regime has been conducted using a Kubo formula, ${ }^{58-60}$ and to the best of our knowledge only one work has taken the approach outlined above to calculate the magnetization directly from the renormalization of the $p f$ spectral function. ${ }^{52}$ Since, however, this latter work contains an error, and as we think that the calculation is instructive, we shall consider this simpler case in some detail before briefly discussing the nonequilibrium case.

\section{Equilibrium magnetization}

At zero-bias voltage the local spin is in equilibrium with the leads, and the $p f$ distribution function reduces to a simple Fermi function. In the Keldysh formalism, this may be viewed as a simple consequence of the KMS (KuboMartin-Schwinger) boundary condition, ${ }^{52}$ which states that in thermal equilibrium $\mathcal{G}_{\gamma \lambda}^{>}(\omega)=-\mathcal{G}_{\gamma \lambda}^{<}(\omega) \exp [(\omega+\lambda) / T]$. The quantum Boltzmann equation (48) may be rewritten as

$$
\Sigma_{\gamma \lambda}^{>}(\omega) \mathcal{G}_{\gamma \lambda}^{<}(\omega)=\Sigma_{\gamma \lambda}^{<}(\omega) \mathcal{G}_{\gamma \lambda}^{>}(\omega),
$$

from which the KMS condition is seen to imply that

$$
\frac{\Sigma_{\gamma \lambda}^{>}(\omega)}{\Sigma_{\gamma \lambda}^{<}(\omega)}=-e^{(\omega+\lambda) / T} .
$$

This can also be verified explicitly from our perturbative expressions for $\Sigma^{>,<}$, by employing the (KMS) condition $1-n_{\gamma \lambda}=n_{\gamma \lambda} \exp [(\lambda-\gamma B / 2) / T] \quad$ (explicitly satisfied by a Fermi function) for the on-shell equilibrium occupation numbers. Inserting (A4) into Eq. (51) shows that indeed 


$$
n_{\gamma \lambda}(\omega)=\frac{1}{e^{(\omega+\lambda) / T}+1},
$$

and when derived in this way, it becomes clear that the KMS condition ensures a highly nontrivial cancellation of interaction corrections in the case of thermal equilibrium. Applying a finite voltage, this condition is violated and the nonequilibrium distribution function will be affected by interactions in the manner which we have described in Sec. III B.

As mentioned earlier, the first-order $p f$ Hartree selfenergy vanishes unless one includes a Zeeman term for the conduction electrons. However, adding such a term,

$$
H_{\text {Zeeman }}^{c e}=B \sum_{\mathbf{k}, \sigma ; \mathbf{k}^{\prime}, \sigma^{\prime} ; \alpha} c_{\alpha \mathbf{k} \sigma^{\dagger}}^{\dagger} \tau_{\sigma \sigma^{\prime}}^{3} c_{\alpha \mathbf{k}^{\prime} \sigma^{\prime}}
$$

to the Hamiltonian, one finds that the self-energy is entirely real and given as

$$
\operatorname{Re}\left[\Sigma_{\gamma}^{(1)}(\omega)\right]=\frac{1}{4 i} J_{\alpha \alpha} \tau_{\sigma \sigma}^{i} \tau_{\gamma \gamma}^{i} \int \frac{d \varepsilon}{2 \pi} \Lambda_{11}^{c d} \underline{G}_{\alpha \sigma}^{d c}(\varepsilon)=\gamma B g_{d} / 2
$$

where the $c e$ Green function now depends on the spin. Note that the Keldysh component of the Hartree self-energy is identically zero, due to the fact that $\operatorname{Re}\left[G_{\alpha \gamma}(\varepsilon)\right]$ is an uneven function. In a case where particle-hole symmetry is broken on an energy scale $\delta$, one obtains a finite contribution of order $J \delta / D$, which can be neglected for large $D$. The only effect of including a Zeeman term for the conduction electrons is therefore a constant shift of the $p f$ energy levels.

The second-order $p f$ self-energy has a real part, which has only a negligible contribution from the term (A6). Without this term, one finds that

$$
\begin{aligned}
\operatorname{Re}\left[\Sigma_{\gamma}^{(2)}(\omega)\right]= & -\frac{g_{\alpha \alpha^{\prime}}^{2}}{32} \theta_{\gamma \gamma^{\prime}} \int_{-D}^{D} d \varepsilon \tanh \left(\frac{\varepsilon+\omega-\mu_{\alpha^{\prime}}}{2 T}\right) \\
& \times \ln \left(\frac{D^{2}}{\left(\varepsilon-\mu_{\alpha}-\gamma^{\prime} B / 2\right)^{2}+T^{2}}\right)
\end{aligned}
$$

which implies that

$$
\operatorname{Re}\left[\Sigma_{\gamma}^{(2)}(-\gamma B / 2)\right] \approx \gamma B g^{2} \ln \frac{D}{T}
$$

and

$$
\partial_{\omega} \operatorname{Re}\left[\Sigma_{\gamma}^{(2)}(\omega)\right] \approx-\frac{3}{2} g^{2} \ln \frac{D}{T}
$$

in the case of $V=0$ and $T \gg B$.

The broadening of the $p f$ energy levels is given by the imaginary part of the self-energy as $\Gamma=i\left(\Sigma^{>}-\Sigma^{<}\right)$, and when neglecting $\Sigma^{<}$, which projects to zero, we end up with $\Gamma=i \Sigma^{>}$which is given by

$$
\Gamma_{\gamma}(\omega)=g^{2} \theta_{\gamma \gamma^{\prime}}\left(\omega+\gamma^{\prime} B / 2\right)\left[1+N\left(\omega+\gamma^{\prime} B / 2\right)\right]
$$

to second order in $g$. This function is highly asymmetric and expanding to first order in $B / T$ one finds the asymptotic behavior

$$
\Gamma_{\gamma}(\omega)= \begin{cases}3 \pi g^{2} \omega, & T \ll \omega \\ 3 \pi g^{2} T\left(1-\frac{\gamma B}{3 T}\right), & -T \ll \omega \ll T \\ 3 \pi g^{2}|\omega| e^{-|\omega| / T}\left(1-\frac{\gamma B}{6 T}\right), & \omega \ll-T,\end{cases}
$$

complemented by the fact that $\Gamma_{\gamma}(\omega)=0$ for $|\omega|>2 D$, since the excitation of particle-hole pairs giving rise to the broadening is limited by the bandwidth. Since $\Gamma$ is essentially constant near the peak of the spectral function, one may approximate $\mathcal{A}_{\gamma}$ in Eq. (49) by a Lorentzian for $\omega \in[-T, T]$ and by $\Gamma_{\gamma}(\omega) / \omega^{2}$ for $|\omega|>T$. Introducing the wave-function renormalization factor

$$
Z_{\gamma}(\omega)=\left|1-\partial_{\omega} \operatorname{Re}\left[\Sigma_{\gamma}(\omega)\right]\right|^{-1}=1-\frac{3}{2} g^{2} \ln \frac{D}{T},
$$

the coherent part of this approximate spectral function integrates to $Z$ in the interval $[-T, T]$. The exponential integral of the negative frequency tail contributes with a number of the order of $g^{2}$, which should be neglected, while the integral from $T$ to $D$ yields exactly $1-Z$, which ensures that this approximate spectral function integrates to 1 .

To find the magnetization, the integral (A2) may now be evaluated using the Boltzmann distribution and this approximate spectral function. The spectral function is centered at a frequency $\omega_{\gamma}$ satisfying the equation $\omega_{\gamma}=-\gamma B / 2$ $+\operatorname{Re}\left[\Sigma_{\gamma}\left(\omega_{\gamma}\right)\right]$, and consequently the integral over $[-T, T]$ contributes a factor of $Z n_{\gamma \lambda}\left(\omega_{\gamma}\right)$, that is,

$$
\begin{gathered}
\int_{-T}^{T} \frac{d \omega}{2 \pi} \frac{\Gamma_{\gamma}}{\left(\omega-\omega_{\gamma}\right)^{2}+\left(\Gamma_{\gamma} / 2\right)^{2}} Z n_{\gamma \lambda}(\omega) \\
\approx Z+\gamma \frac{B}{2 T}\left(1-g-\frac{7}{2} g^{2} \ln \frac{D}{T}\right),
\end{gathered}
$$

to first order in $B / T$. Since the distribution function falls off exponentially for $\omega \gg T$, the integral over $[T, D]$ is negligible. On the negative frequency tail, the spectral function decays exponentially, but this is compensated in the integral by the exponential increase of the Boltzmann distribution, and one finds that

$$
\int_{-D}^{-T} \frac{d \omega}{2 \pi} \frac{\Gamma_{\gamma}}{\omega^{2}} n_{\gamma \lambda}(\omega) \approx 1-Z-\gamma \frac{B}{4 T} g^{2} \ln \frac{D}{T}
$$

Adding up Eqs. (A14) and (A15) and inserting in Eq. (A1), one ends up with the magnetization

$$
M=\frac{B}{2 T}\left(1-g-4 g^{2} \ln \frac{D}{T}\right),
$$


to which one may finally add the corresponding induced spin polarization of the conduction electrons to obtain the total magnetization

$$
\left\langle S^{z}+s_{L}^{z}+s_{R}^{z}\right\rangle-\left\langle s_{L}^{z}+s_{R}^{z}\right\rangle_{0}^{\text {Pauli }} \approx \frac{B}{4 T}\left(1-2 g-4 g^{2} \ln \frac{D}{T}\right) .
$$

Here the $z$ component of the total spin of lead $\alpha$ has been introduced as

$$
s_{\alpha}^{z}=\sum_{\mathbf{k}, \sigma ; \mathbf{k}^{\prime}, \sigma^{\prime} ; \alpha} c_{\alpha \mathbf{k} \sigma}^{\dagger} \tau_{\sigma \sigma^{\prime}}^{3} c_{\alpha \mathbf{k}^{\prime} \sigma^{\prime}},
$$

and the $p f$ and ce $g$ factors are assumed to be equal. This result matches the high-temperature expansion of the exact Bethe ansatz solution as it should. ${ }^{1,61}$

In Ref. 51, the normalization of the approximate spectral function is demonstrated just like here. In calculating the magnetization, however, the contribution (A15) was not included and the $Z$ factor was argued to be canceled by the same factor appearing in the denominator of Eq. (A1). Altogether, this error leads to a prefactor of 2 instead of 4 in front of the $g^{2} \ln (D / T)$-term in Eq. (A16), which destroys the correspondence with the exact result.

\section{Nonequilibrium magnetization}

As demonstrated in the equilibrium case, the magnetization is renormalized in a delicate balance between shifts and broadening of the $p f$ energy levels, i.e., between the influence of $\operatorname{Re}[\Sigma]$ on the coherent part, and of $\Gamma$ on the incoherent tails of the spectral function. In the case of finite bias voltage, however, the renormalization of the distribution function becomes important.

At finite voltage, there are logarithmic corrections to $\Sigma<$ and $\Sigma^{>}$, which no longer cancel in Eq. (51), and the magnetization now exhibits the stronger renormalization by factors of $g \ln (D)$ rather than $g$ and $g^{2} \ln (D)$. To properly describe the crossover to equilibrium, as $T$ becomes greater than $V$, one should include these subleading corrections deriving from the renormalization of the $p f$ spectral function. This can be done in much the same way as above, as long as care is taken to separate the coherent part of the spectral function from the incoherent tails at either $T$ or $V$, so as to ensure normalization of the total spectral weight. When $T$ is increased beyond $V$, the $g \ln (D)$ corrections get less and less important.

It should be emphasized that, as mentioned already in Sec. IV, the effects of shifts and broadening, discussed in this appendix, affect only the observable magnetization and have no influence on our result for the leading logarithmic corrections to the current.
${ }^{1}$ A.C. Hewson, The Kondo Problem to Heavy Fermions (Cambridge University Press, Cambridge, 1993).

${ }^{2}$ J. Kondo, Prog. Theor. Phys. 32, 37 (1964).

${ }^{3}$ N. Andrei, Phys. Rev. Lett. 45, 379 (1980); Phys. Lett. 87A, 299 (1982).

${ }^{4}$ K.G. Wilson, Rev. Mod. Phys. 47, 773 (1975); H.R. Krishnamurthy, J.W. Wilkins, and K.G. Wilson, Phys. Rev. B 21, 1003 (1980)

${ }^{5}$ P.W. Anderson, J. Phys. C 3, 2436 (1970).

${ }^{6}$ L. Glazman and M. Raikh, JETP Lett. 47, 452 (1988).

${ }^{7}$ T.K. Ng and P.A. Lee, Phys. Rev. Lett. 61, 1768 (1988).

${ }^{8}$ D. Goldhaber-Gordon, Hadas Shtrikman, D. Mahalu, David Abusch-Magder, U. Meirav, and M.A. Kastner, Nature (London) 391, 156 (1998).

${ }^{9}$ S.M. Cronenwett, T.H. Oosterkamp, and L.P. Kouwenhoven, Science 281, 540 (1998).

${ }^{10}$ J. Schmid, J. Weis, K. Eberl, and K. von Klitzing, Physica B 258, 182 (1998).

${ }^{11}$ J. Nygård, D.H. Cobden, and P.E. Lindelof, Nature (London) 408, 342 (2000).

${ }^{12}$ W.G. van der Wiel, S. De Franceschi, T. Fujisawa, J.M. Elzerman, S. Tarucha, and L.P. Kouwenhoven, Science 289, 2105 (2000).

${ }^{13}$ L.Y.L. Shen and J.M. Rowell, Phys. Rev. 165, 566 (1968).

${ }^{14}$ P. Nielsen, Phys. Rev. B 2, 3819 (1970).

${ }^{15}$ J. Appelbaum and L.Y. Shen, Phys. Rev. B 5, 544 (1972).

${ }^{16}$ R.H. Wallis and A.F.G. Wyatt, J. Phys. C 7, 1293 (1974).

${ }^{17}$ S. Bermon, D.E. Paraskevopoulos, and P.M. Tedrow, Phys. Rev. B 17, 2110 (1978).

${ }^{18}$ R. Magno and J.G. Adler, Phys. Rev. B 15, 1744 (1977).
${ }^{19}$ E.L. Wolf and D.L. Losee, Phys. Rev. B 2, 3660 (1970).

${ }^{20}$ E.L. Wolf, in Solid State Physics, edited by H. Ehrenreich, F. Seitz, and D. Turnbull (Academic, New York, 1975), Vol. 30, p. 1.

${ }^{21}$ J. Appelbaum, Phys. Rev. Lett. 17, 91 (1966).

${ }^{22}$ J. Appelbaum, Phys. Rev. 154, 633 (1967).

${ }^{23}$ P.W. Anderson, Phys. Rev. Lett. 17, 95 (1966).

${ }^{24}$ D.L. Losee and E.L. Wolf, Phys. Rev. Lett. 23, 1457 (1969).

${ }^{25}$ T. Ivezić, J. Magn. Magn. Mater. 15-18, 933 (1980).

${ }^{26}$ J. Sólyom and A. Zawadowski, Phys. Kondens. Mater. 7, 325 (1968); 7, 342 (1968).

${ }^{27}$ J. Appelbaum and W.F. Brinkman, Phys. Rev. B 2, 907 (1970).

${ }^{28}$ A. Zawadowski, Phys. Rev. 163, 341 (1967).

${ }^{29}$ J. Appelbaum and W.F. Brinkman, Phys. Rev. 186, 464 (1969).

${ }^{30}$ T. Ivezić, J. Phys. C 8, 3371 (1975).

${ }^{31}$ C. Caroli, R. Combescot, P. Nozieres, and D. Saint-James, J. Phys. C 4, 916 (1971).

${ }^{32}$ E.L. Wolf, Principles of Electron Tunneling Spectroscopy (Oxford University Press, Oxford, 1985).

${ }^{33}$ Y. Meir, N.S. Wingreen, and P.A. Lee, Phys. Rev. Lett. 70, 2601 (1993).

${ }^{34}$ N.S. Wingreen and Y. Meir, Phys. Rev. B 49, 11040 (1994).

${ }^{35}$ M.H. Hettler, J. Kroha, and S. Hershfield, Phys. Rev. Lett. 73, 1967 (1994); Phys. Rev. B 58, 5649 (1998).

${ }^{36}$ P. Nordlander, M. Pustilnik, Y. Meir, N.S. Wingreen, and D.C. Langreth, Phys. Rev. Lett. 83, 808 (1999).

${ }^{37}$ M. Plihal, D.C. Langreth, and P. Nordlander, Phys. Rev. B 61, R13 341 (2000).

${ }^{38}$ M. Krawiec and K.I. Wysokinski, Phys. Rev. B 66, 165408 (2002). 
${ }^{39}$ N. Sivan and N.S. Wingreen, Phys. Rev. B 54, 11622 (1996).

${ }^{40}$ Y. Goldin and Y. Avishai, Phys. Rev. Lett. 81, 5394 (1998); Phys. Rev. B 61, 16750 (2000).

${ }^{41}$ A. Kaminski, Yu.V. Nazarov, and L.I. Glazman, Phys. Rev. Lett. 83, 384 (1999); Phys. Rev. B 62, 8154 (2000).

${ }^{42}$ A. Schiller and S. Hershfield, Phys. Rev. B 51, 12896 (1995); . 58, 14978 (1998); K. Majumdar, A. Schiller, and S. Hershfield, ibid. 57, 2991 (1998).

${ }^{43}$ V.J. Emery and S. Kivelson, Phys. Rev. B 46, 10812 (1992).

${ }^{44}$ R.M. Konik, H. Saleur, and A.W.W. Ludwig, Phys. Rev. Lett. 87, 236801 (2001); Phys. Rev. B 66, 125304 (2002).

${ }^{45}$ A. Rosch, J. Paaske, J. Kroha, and P. Wölfle, Phys. Rev. Lett. 90, 076804 (2003).

${ }^{46}$ J. Appelbaum, J.C. Phillips, and G. Tzouras, Phys. Rev. 160, 554 (1967)

${ }^{47}$ Y. Nagaoka, Phys. Rev. 138, A1112 (1965).

${ }^{48}$ A.A. Abrikosov, Physics 2, 5 (1965); 2, 61 (1965).

${ }^{49}$ H. Suhl, Phys. Rev. 138, A515 (1965); H. Suhl and D. Wong, Physics 3, 17 (1967).

${ }^{50}$ A. Rosch, J. Paaske, J. Kroha, and P. Wölfle (unpublished).
${ }^{51}$ A. Zawadowski and P. Fazekas, Z. Phys. 226, 235 (1969).

${ }^{52}$ J. Rammer and H. Smith, Rev. Mod. Phys. 58, 323 (1986).

${ }^{53}$ L.P. Kadanoff and G. Baym, Quantum Statistical Mechanics (Benjamin, New York, 1962).

${ }^{54}$ D.C. Langreth, in Linear and Nonlinear Electron Transport in Solids, edited by J.T. Devreese and E. Van Doren (Plenum, New York, 1976).

${ }^{55}$ O. Parcollet and C. Hooley, Phys. Rev. B 66, 085315 (2002).

${ }^{56}$ A. Rosch, J. Kroha, and P. Wölfle, Phys. Rev. Lett. 87, 156802 (2001).

${ }^{57}$ J. Paaske, A. Rosch, J. Kroha, and P. Wölfle, cond-mat/0401180 (unpublished).

${ }^{58}$ K. Yosida and A. Okiji, Prog. Theor. Phys. 34, 505 (1965).

${ }^{59}$ W. Brenig, J.A. Gonzalez, W. Götze, and P. Wölfle, Z. Phys. 235, 52 (1970).

${ }^{60}$ A. Okiji, A. Kato, and H. Shiba, Suppl. Prog. Theor. Phys. 46, 182 (1970).

${ }^{61}$ N. Andrei, K. Furuya, and J.H. Lowenstein, Rev. Mod. Phys. 55, 331 (1983). 